در اسة تحليلية للموقف الحالى لإتتاج أهم أصناف الموالح فى مصر

سعاد عبد اللطيف موسى محمد ، شهيزة محمد رضا ابر اهيم

معهز بحوث الافتصاد الزراعي - مركز البحوث الزراعية

المستخلص

تعتبر الموالح من أهم أنواع الفاكهة إنتشاراً فى معظم المحافظات داخل

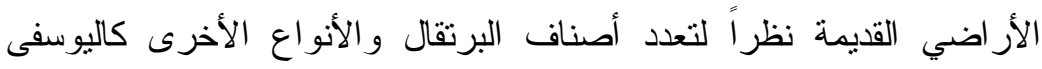

و الليمون المالح حيث تأتي فى المركز الأول من حيث المساحة الكلية وقيمة

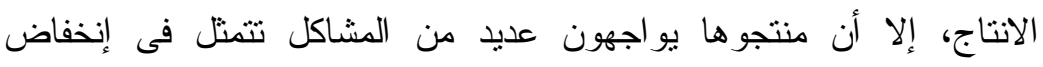

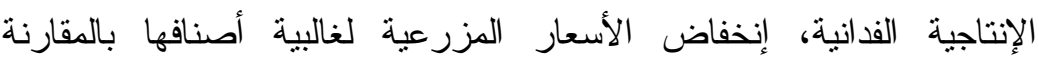

بنظير اتها بالأر اضي الجديدة، وكذلك إرتفاع التكاليف الإنتاجية مما يترتب عليه

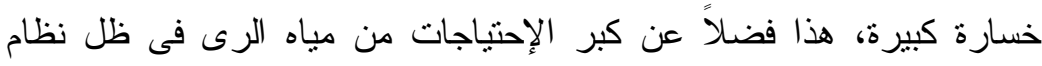

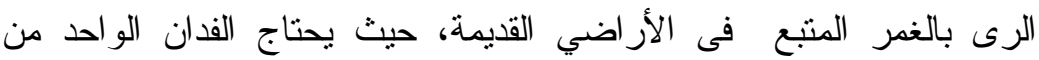

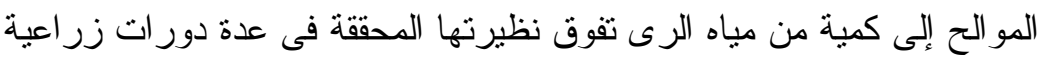

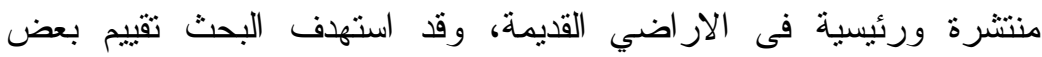
السياسات الزراعية المرتبطة بأهم أصناف الموالح فى ظلى ظل محدودية موردي ولئي الارض و المياه. وأوضح البحث أن أعلى إنتاجية من الوحدة الأرضية

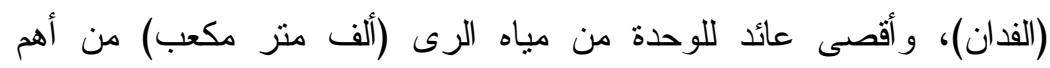

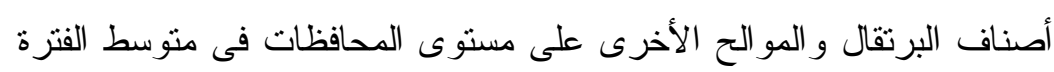

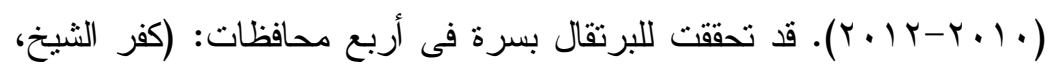

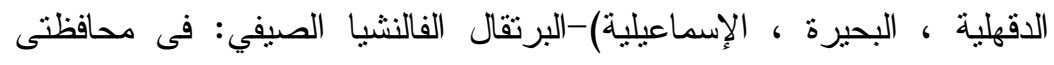
الإسماعيلية و البحيرة -البرتقال البلاي الطعم: فى محافظات الغربية، البحيرة،

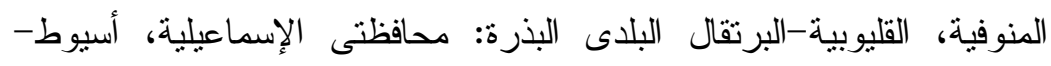

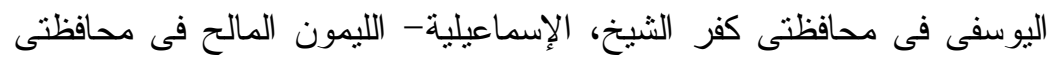

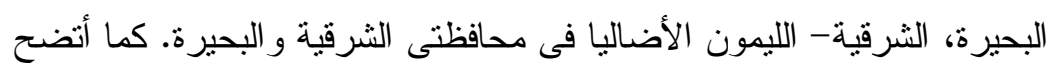
أن التكاليف الإنتاجية الكلية للفدان المثر من الموالح فئ الأر اضي القديمة فى الفى

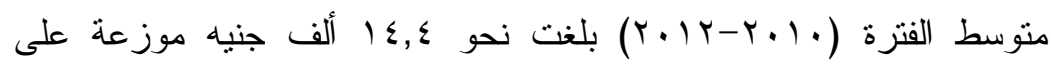

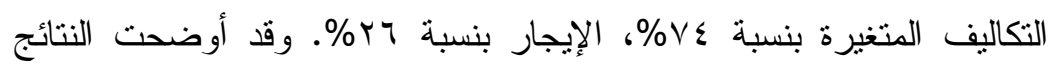

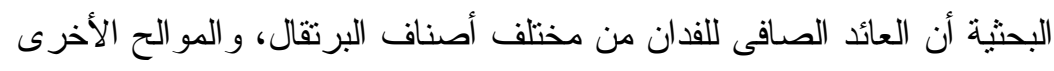

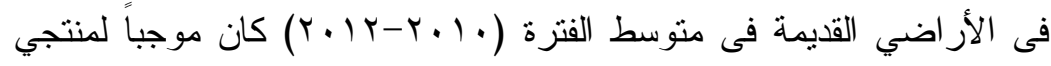
الليمون الأضاليا والليمون المالح، حيث قدر بنحو 
على الترنيب، فى حين تبين أن هذا العائد كان سالباً للمنتجين فى جميع أصناف البرتقال حيث تر اوح ما بين حد أدنى فى البرتقال الفالنشيا الصيفي لئي

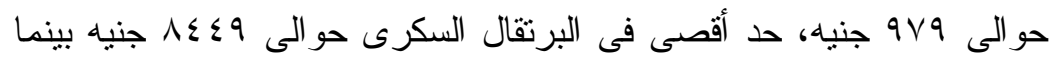

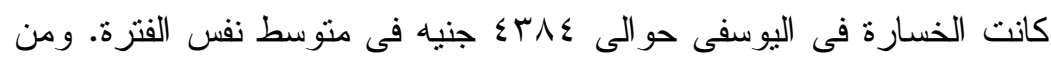

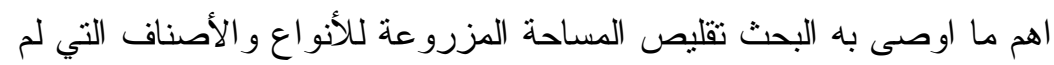
تحقق جدارة إنتاجية فدانية وأكبر عائد من وحدة مياه الرى (الألف متر مكعب) وهى البرتقال بسرة فى محافظتى المنوفية والثرقية، البرتقال الفالنشيا

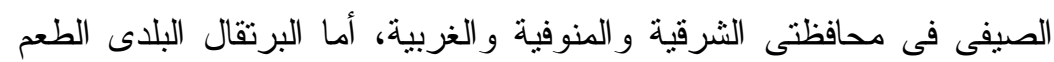

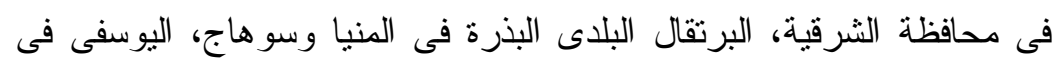

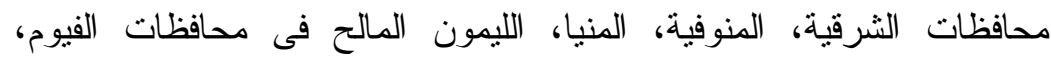

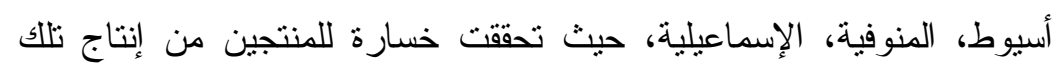
الأصناف بدرجة واضحة، وإحلال زراعة الإحاصيل التقليدية الحقلية

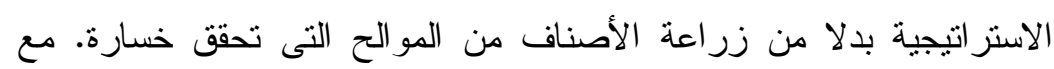
التوسع فى إستخدام العمل الآلى وذلك يسهم فى خفض التها لتكاليف الإنتاجية الكلية الفدانية لأصناف المو الح فى الأر اضي القديمة مما يؤدي إلى زيادة فى العو ائد الصافية للمو الح.

\section{مقدمة}

تعثبر المو الح من أهم أنو اع الفاكهة إنتشار اً فى معظم المحافظات داخل الأر اضي القديمة نظر اً لتعدد أصناف البرتقال و الأنواع الأخرى كاليوسفى و الليمون المالح، حيث قدرت الفئ مساحتها الكلية وقيمة

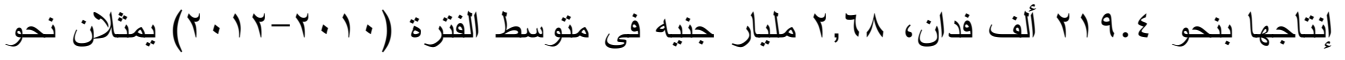

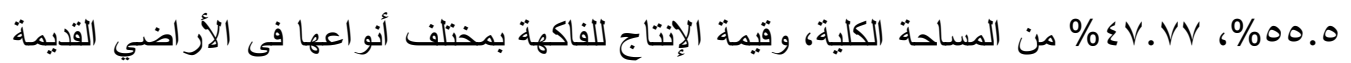

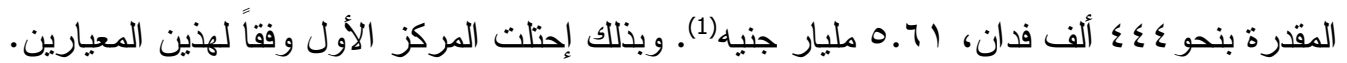

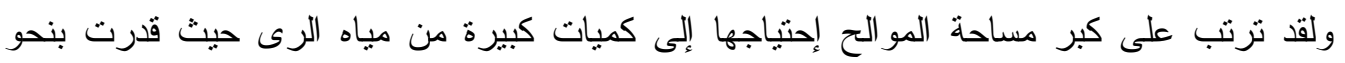

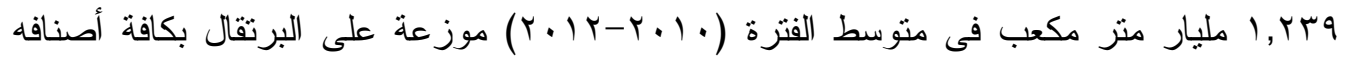

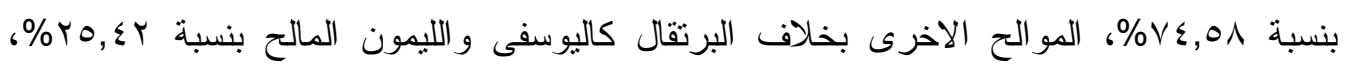

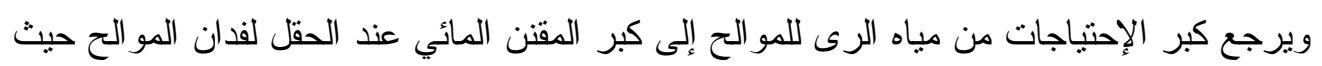

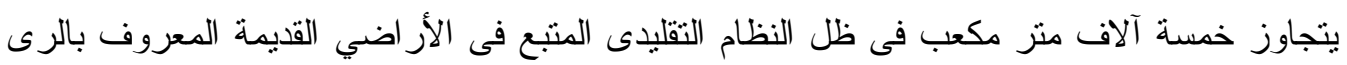

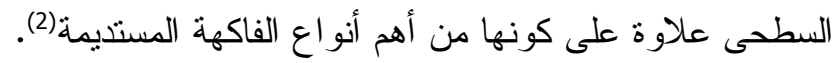
مشكلة البحث

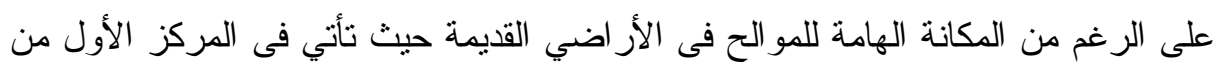

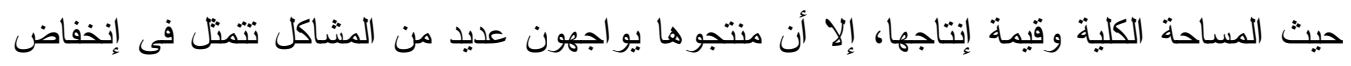

(1) الجهاز المركزى للتعبئة العامة والاحصاء، النشرة السنوية للموارد المائية و الرى، أعداد مختلفة. (2)عاطف محمد إبر اهيم (دكتور)، محمد نظيف حجاج (دكتور)، الموالح زراعتها ورعايتها وإنتاجه، الطبعة الاولى، الناشر منشأة 
الإتتاجية الفدانية، إنخفاض الأسعار المزرعية لغالبية أصنافها بالمقارنة بنظير اتها بالأر اضي الجديدة،

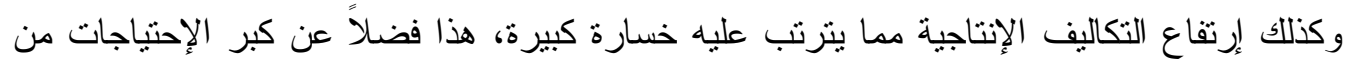

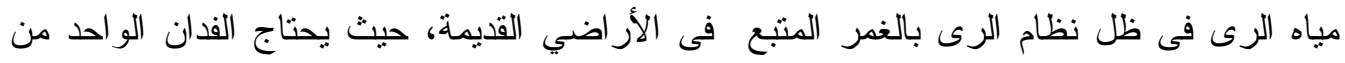

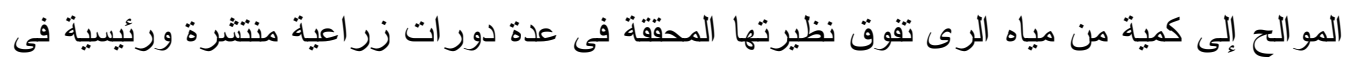
الار اضي القديمة.

\section{هدف البحث}

يستهدف البحث بصفة عامة تقييم بعض السياسات الزر اعية المرتبطة بأهم أصناف المو الح

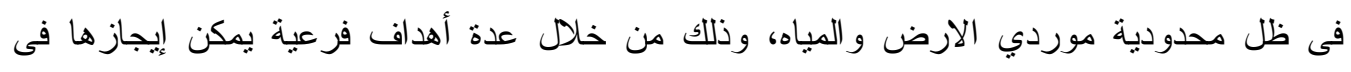
العناصر النالية:

1 - تحديد الأهمية الاقتصادية للمو الح بين أنواع الفاكهة المنتجة فى الأر اضي القديمة.

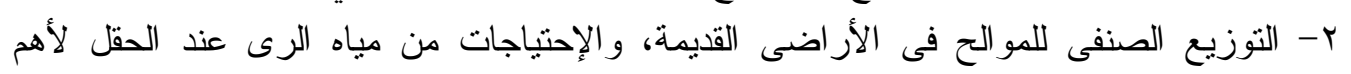
أصناف المو الح.

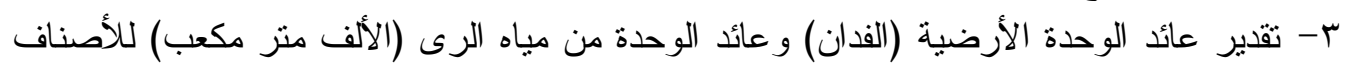

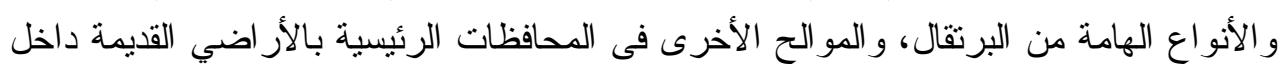

$$
\text { الو ادي. }
$$

ع- - التعرف على المحافظات التى إنخفضت بها الجدارة الانتاجية و المحافظات الاعلى إنتاجية فدانية

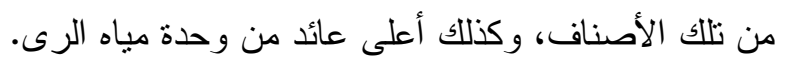

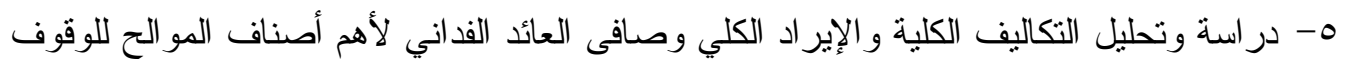

\section{الأسلوب البحثى ومصادر البيانات}

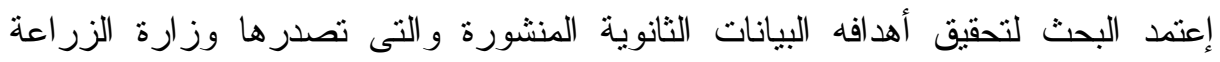

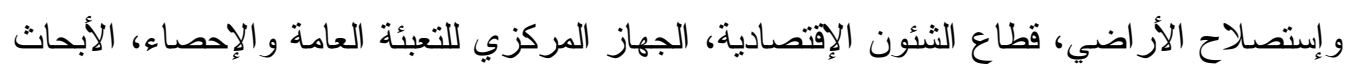

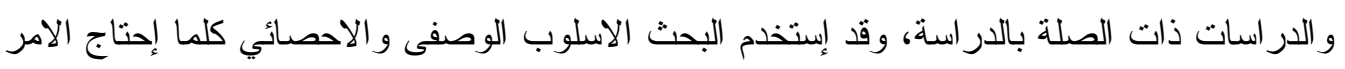
الى ذلك للحصول على النتائج البحثية.

\section{النتائج البحثية ومناقشتها}

مكانة الموالح بين أنواع الفاكهة المنتجة فى الأراضي القديمة:

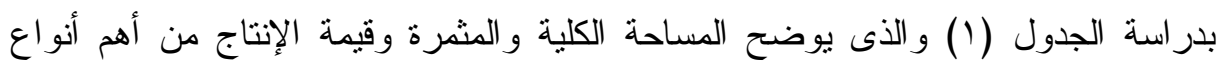

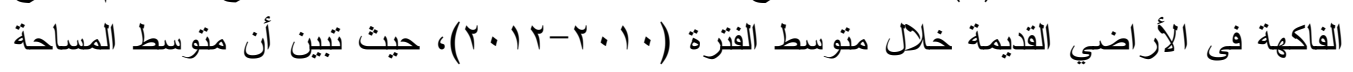

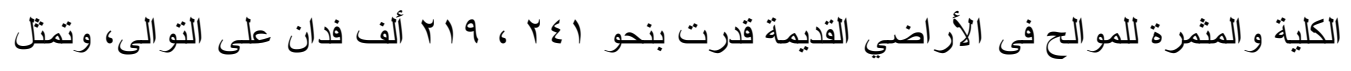

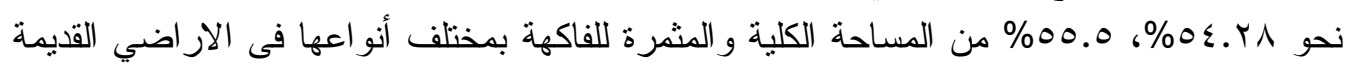

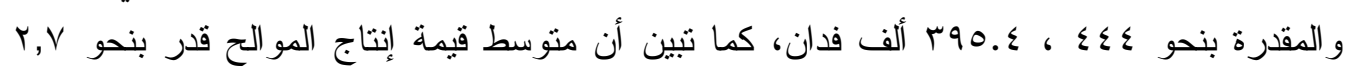

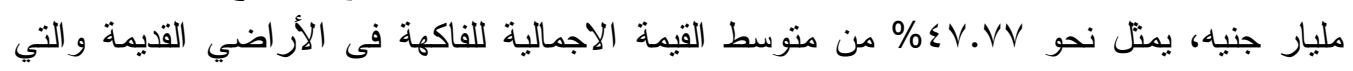

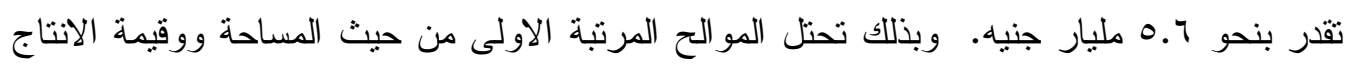

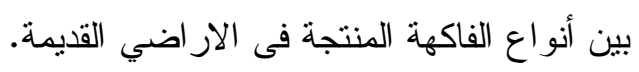


جدول (1) المساحة الكلية و المثرة وقيمة الإنتاج لأهم أنواع الفاكهة فى الأر اضي القديمة

\begin{tabular}{|c|c|c|c|c|c|c|c|c|}
\hline الترتيب & $\%$ & قليمة الانتاج & التزتيب & $\%$ & 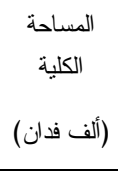 & $\%$ & 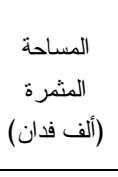 & أهم الأنو اع \\
\hline 1 & $\varepsilon V . V V$ & (YMA & 1 & $0 \xi . r \Lambda$ & $r \leqslant 1$ & 00.0 & $r \mid q, \varepsilon$ & مو الح \\
\hline$\varepsilon$ & 15.94 & VAr & $r$ & $Y \varepsilon . Y Y$ & 11. & $r$ r.r & $a r$ & مانجو \\
\hline$r$ & $1 \varepsilon . \cdot A$ & vq. & $r$ & $\mid r . \Lambda \varepsilon$ & or & $1 r . r$ & or & عiع \\
\hline$r$ & YE.TY & $1 r 09$ & $\varepsilon$ & A.11 & $r 4$ & 1.1 & $r$ & موز \\
\hline- & $1 \ldots$ & Tाו & - & $1 .$. & $\varepsilon \varepsilon \varepsilon$ & - & $r 90 . \varepsilon$ & الاجمالى \\
\hline
\end{tabular}

تضم الفاكهة أنو اع أخرى منل التفاح، الكمثرى، الجو افة، البرقوق، وغير ها.

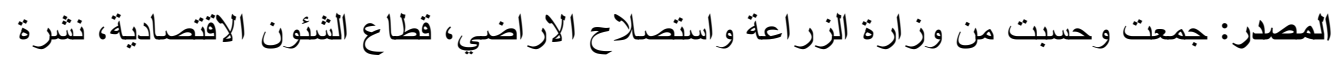
الاحصاءات الزر اعية ، أعداد متقرقة. التوزيع الصنفى للبرتقال فى الأراضي القديمة:

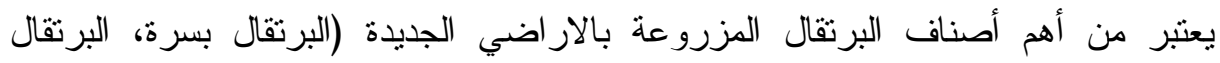

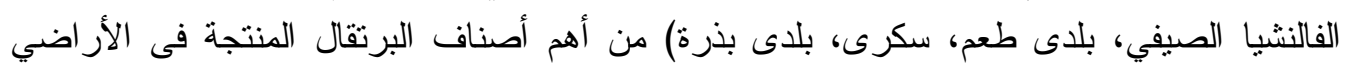

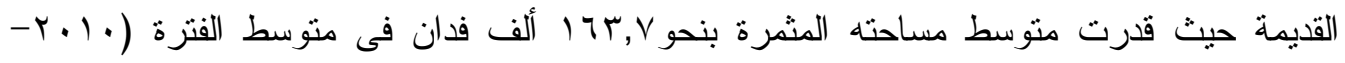

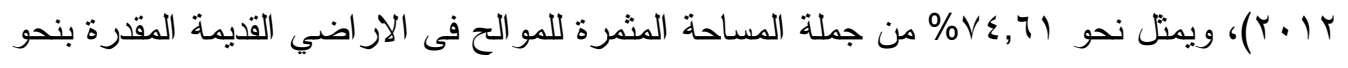

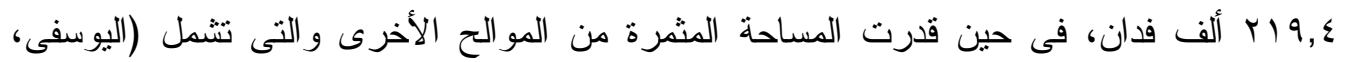

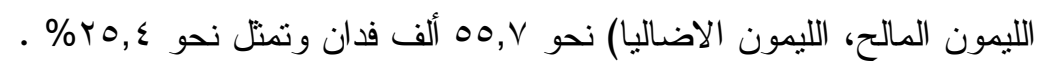

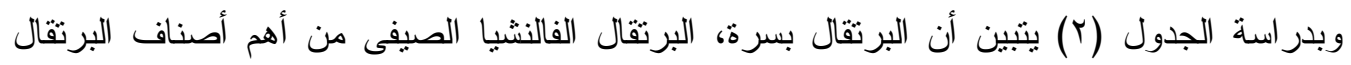

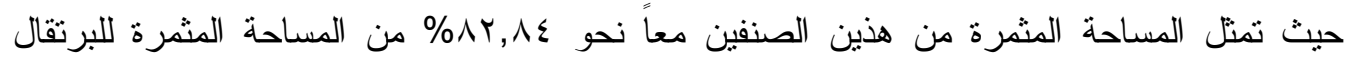

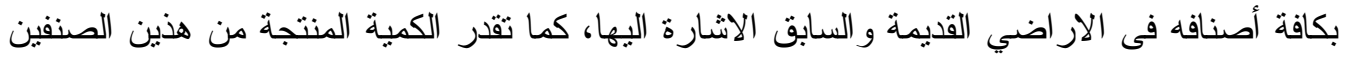

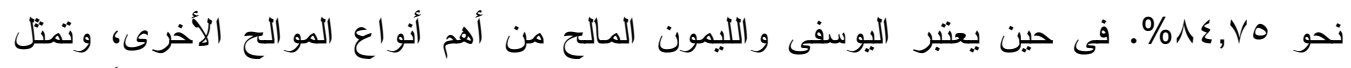

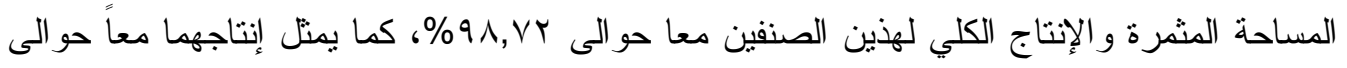

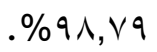

الإحتياجات المائية وفقاً للمقنن الحقلى لأهم أصناف البرتقال فى الأراضي القديمة:

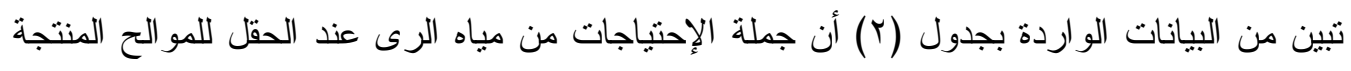

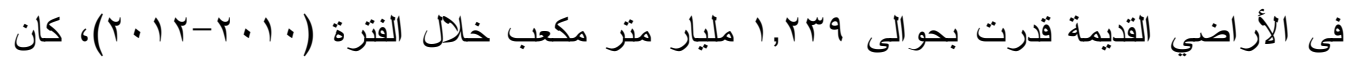

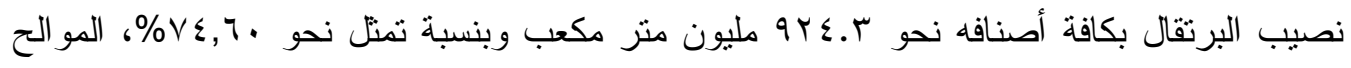

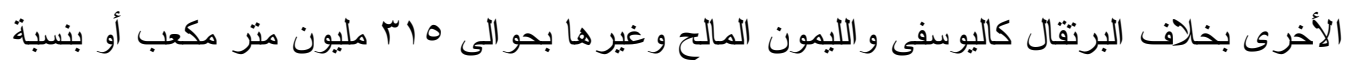

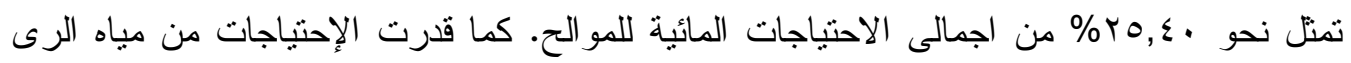

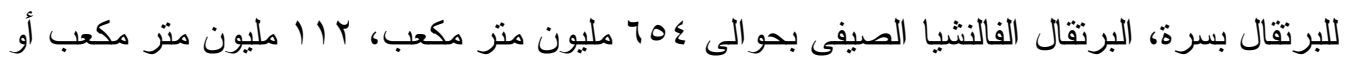

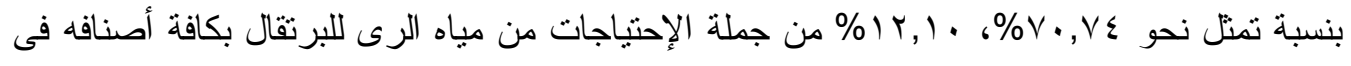

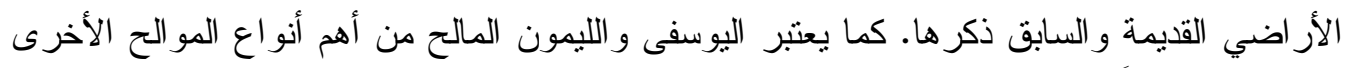

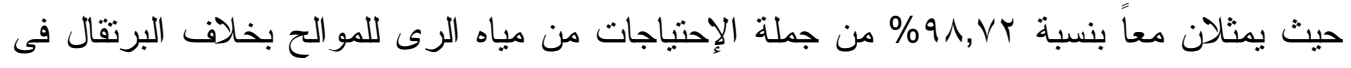

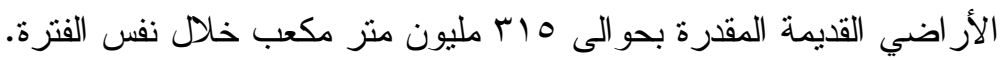


جدول (Y) المساحة والانتاج وقيمة الانتاج و الاحتياجات المائية

\begin{tabular}{|c|c|c|c|c|c|c|c|c|}
\hline$\%$ & مل الإحنياجات & $\%$ & قليمة الإنتاج & $\%$ & (الفن طن النتاج الكلي & $\%$ & (المثن فدرة & الأنو اعو والأصناف \\
\hline$v \cdot, v \varepsilon$ & $70 r, A 1$ & V४, rq & $1 \leq \varepsilon,, 1 \leqslant$ & $V 1, Y v$ & $11 \Gamma \cdot, r \mu$ & $v \cdot, v \varepsilon$ & 110,1 & بسرة \\
\hline rr,. & $111, \Delta r$ & $1 \varepsilon, \cdot v$ & rMo,rT & $\mid r, \varepsilon \lambda$ & rIr,V. & $\mid r, 1$. & $|9, \Lambda|$ & فالنشيا صبفي \\
\hline$\lambda, 0 \leqslant$ & $V \wedge, q \varepsilon$ & $\{, 09$ & $\Delta 7,07$ & $\gamma, \varepsilon 1$ & $11 Y, 00$ & $\lambda, 0 \leqslant$ & 15,91 & بلاي طع \\
\hline$\varepsilon, 7$ & \&r, Tr & $r, v_{0}$ & $01, v \lambda$ & $\varepsilon, r_{4}$ & $79, Y)$ & $\varepsilon, 71$ & $r, 00$ & سكرى ( سكر \\
\hline r,vo & $r\{, 77$ & $r, \cdot \varepsilon$ & $r_{\lambda, \Sigma, \imath}$ & $r, r Y$ & $01, \lambda \varepsilon$ & r,vo & $7,1 \varepsilon$ & بلاب بذرة \\
\hline$\ldots$ & $q r \varepsilon, r q$ & $1 \ldots$ & $\mid \Lambda \Lambda 0, Y \leqslant$ & $\ldots$ & 1010,10 & $\ldots$ & $17 r, v 1$ & جملة البرتقال \\
\hline $7 r, 01$ & $199, \vee 9$ & $\varepsilon \varepsilon, r q$ & rot,ru & Tr,or & $r v q, r Y$ & $7 r, 01$ & ro,rq & يوسفي \\
\hline$r 0, r$ & $11 \cdot, \mathrm{rA}$ & $0 \varepsilon, r q$ & $\varepsilon r, r$. & $r u, r v$ & $1 \pi r, . r$ & $r_{0, r}$ & $19, \pi r$ & ليمون مالح \\
\hline 海, & 1,00 & 列, & $7,7 \mathrm{~V}$ &., 07 & $r, 01$ &.,$\leqslant 9$ & . Y, & ليهون أضاليا \\
\hline $1 \ldots$ & $r \mid \varepsilon, 0 \wedge$ & $\ldots$ & ห९१,.० & $1 .$. & $\varepsilon \leqslant 7, V_{0}$ & $1 \ldots$ & $00, \mathrm{~V}$ & جملة المو الح الاخرى \\
\hline- & Ir $\mathrm{r} \lambda, \mathrm{N}$ & - & rדגו,זq & - & T.Tr,T. & - & $r \mid q, \xi$ & جملة المو الح \\
\hline
\end{tabular}

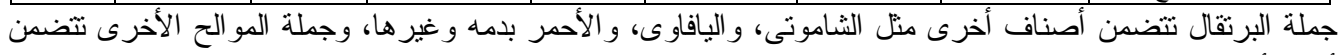

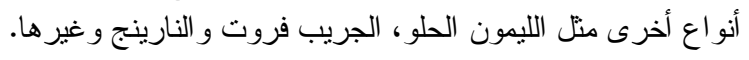

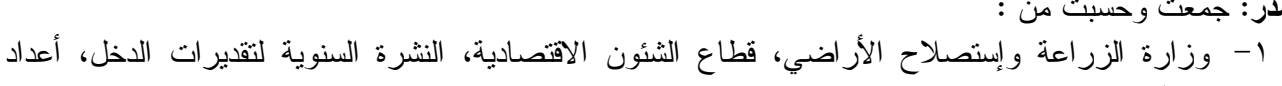

مختلفة.

r- الجهاز المركزى للنعبئة العامة والإحصاء، النشرة السنوية للرى و الموارد، (أعداد مختلفة).

\section{عائد الوحدة الأرضية والإروائية لأهم أنواع الموالح فى أهم المحافظات:}

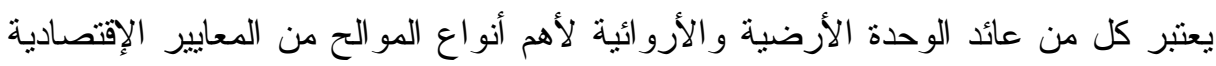

التى يمكن من خلالها ترنيب المحافظات وفقاً لهذين المعيارين.

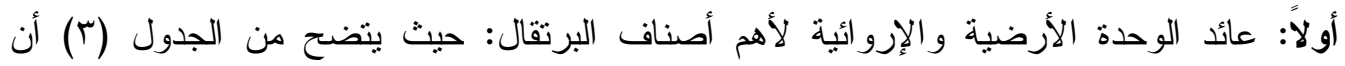

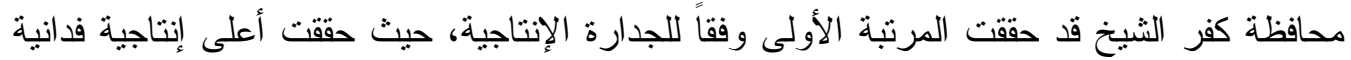

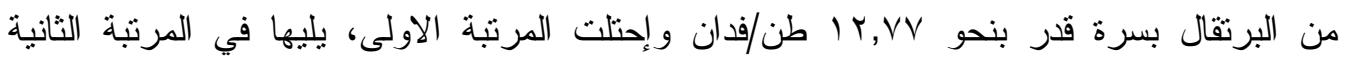

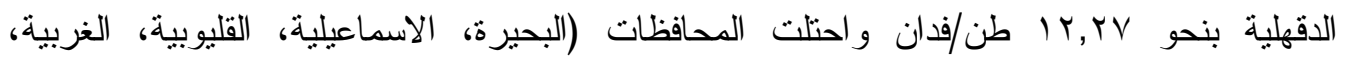

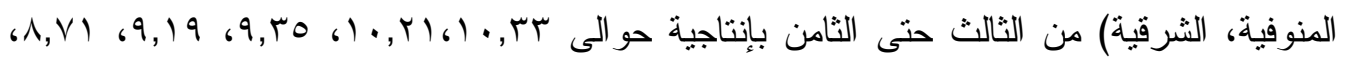

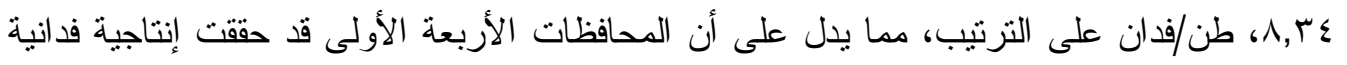

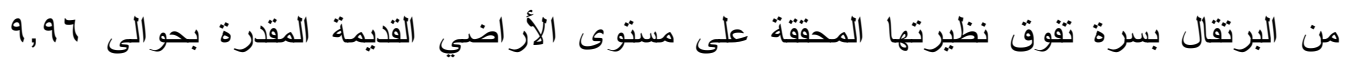

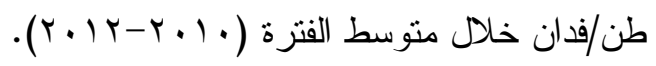

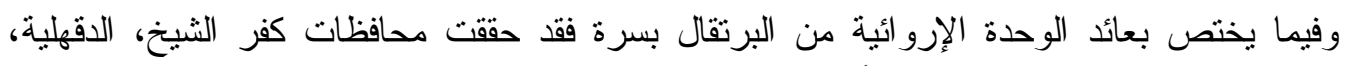

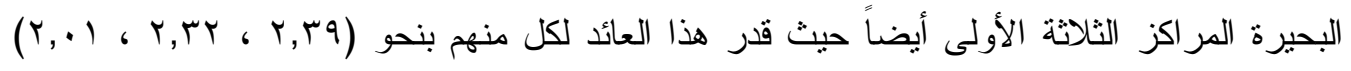

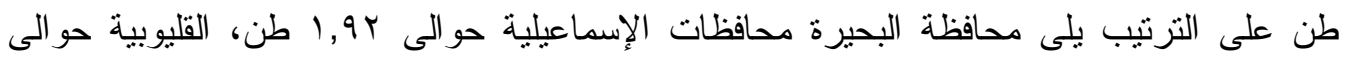

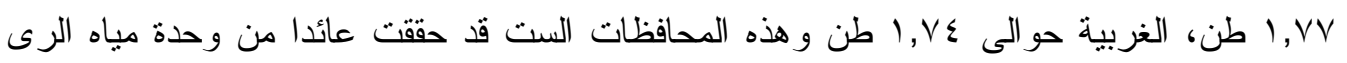

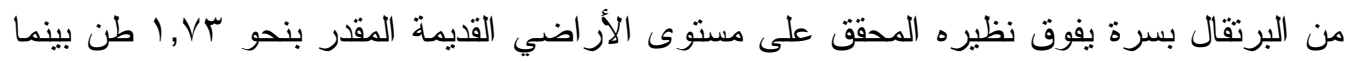

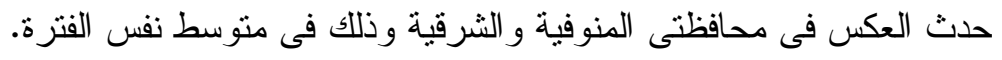




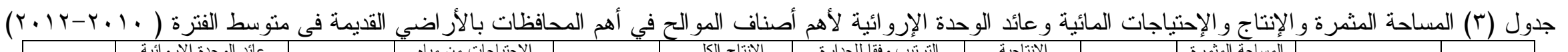

\begin{tabular}{|c|c|c|c|c|c|c|c|c|c|c|c|}
\hline \multirow[t]{2}{*}{ التزتيب } & عائد الوحدة الإروائية & \multirow[t]{2}{*}{$\%$} & الإحتياجات من مياه & \multirow[t]{2}{*}{$\%$} & الإنتاج الكلي & \multirow[t]{2}{*}{ الترتيب وفقا للجدارة } & الإِنتاجية & \multirow[t]{2}{*}{$\%$} & المساحة المثرة & \multirow[t]{2}{*}{ المحافظات } & \multirow[t]{2}{*}{ الصنف } \\
\hline & 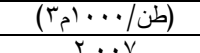 & & $\left.\left(r_{p}\right) \cdots\right)$ & & $\frac{}{(ط)}$ & & (ط) & & 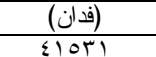 & & \\
\hline$\frac{r}{0}$ & $\begin{array}{l}r, \cdots v \\
1, v 74\end{array}$ & $\frac{r r, r}{r \mu, 1 \Lambda}$ & $\begin{array}{l}\frac{1977 \varepsilon}{1010 \% .} \\
\end{array}$ & $\frac{r q, \cdot 1}{r r, 7 \uparrow}$ & $\frac{\varepsilon \varepsilon \cdot \Lambda \mid r}{\text { rTVTIA }}$ & $\frac{r}{0}$ & $\frac{(1, T) \leq}{q, r \circ Y}$ & $\frac{r 0, \wedge T}{r \varepsilon, Y\}}$ & $\frac{\text { Elorl }}{\text { YN4TY }}$ & القلبيزة & \multirow{8}{*}{ برتقال } \\
\hline V & $1, T \leqslant V$ & $1 r, 91$ & 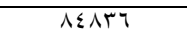 & $|r, r|$ & IrqVIr & V & $\lambda, \vee \backslash \varepsilon$ & $1 T, \wedge 0$ & $17 . r \varepsilon$ & المنوفية & \\
\hline$\Lambda$ & $1,0 \mathrm{VT}$ & 1,19 & orora & $V, \varepsilon 0$ & AEY.r & 1 & $\Lambda, r \leqslant \varepsilon$ & $\Lambda, Y)$ & $1, .91$ & الشرقية & \\
\hline 7 & $1, V \varepsilon 1$ & $0, T V$ & $r v \cdot \Lambda$. & $0, v 1$ & $7 \leqslant 0$ \% & 7 & $9,19 \mathrm{~V}$ & $7, .7$ & $V . I V$ & الغربية & \\
\hline$\varepsilon$ & $1,9 Y 1$ & r,OY & 170.1 & $r, \lambda)$ & rIV.\& & $\varepsilon$ & $T \cdot, r) \leqslant$ & r,79 & $r 1 . \varepsilon$ & الإسماعيلية & \\
\hline 1 & T,rqV & $r, \leqslant 9$ & 174.0 & $r, \leqslant 7$ & $r 9.91$ & 1 & $|r, Y Y|$ & $T, T \leqslant$ & $r \cdot 7)$ & كفر الثيخ & \\
\hline$r$ & T,rIA & $r, \leqslant \uparrow$ & $17.8 V$ & $r, r$. & TVYTY & $r$ & TY,YYT & T,T & $r . r u$ & الدقهلية & \\
\hline- & $1, \vee \times 9$ & $1 \ldots$ & 70rA.V & $1 \ldots$ & $11 r . r 17$ & - & 9,97 & $1 \ldots$ & 1101. & جملة & \\
\hline 7 & $1, \varepsilon \vee 7$ & $r V, \varepsilon Y$ & $r .74$. & $Y, I V$ & EOY $\leqslant Y$ & 7 & $\lambda, Y \neg \lambda$ & $Y V, T Y$ & $0 \leqslant V Y$ & الشرقية & \multirow{7}{*}{ فالنشيال } \\
\hline 1 & $r, I V 4$ & $Y \cdot, 10$ & $T r .9 T$ & $r \xi, \Gamma Y$ & $V T r \leqslant q$ & 1 & $T V, T V$. & $Y \cdot, 97$ & $\{101$ & الاسماعيلية & \\
\hline 0 & 1,070 & $17, .9$ & IV97\& & 15,10 & YAll. & 0 & $\lambda, \vee \backslash 1$ & 17,19 & YYYV & المنوفية & \\
\hline$r$ & $r, \ldots 1$ & 11,01 & TrAYT & $T r, 1 \cdot$ & ro100 & $\bar{r}$ & $11,19 \mathrm{~V}$ & 11,74 & rr.q & البحيرة & \\
\hline$r$ & $1,7) \mathrm{V}$ & $1 \cdot, 9 \mathrm{~V}$ & IrYTA & $9, Y \wedge$ & 19NTr & $r$ & $\Lambda, 99 \lambda$ & $11,1 r$ & Yr. & القليو بية & \\
\hline$\varepsilon$ & 1,071 & 0,99 & 7791 & $\varepsilon, 9 Y$ & 1.0 .7 & $\varepsilon$ & $\lambda, \vee \leqslant$. & $7, \cdot V$ & IT.Y & الغربية & \\
\hline- & 1,911 & $1 \ldots$ & IllAYO & $1 \ldots$ & r)Tr99 & - & $1 \cdot, \vee \vee 9$. & $1 \ldots$ & $19 \lambda .7$ & جملة & \\
\hline 0 & $1, \varepsilon$. & $r \cdot, V_{0}$ & $17 r A$. & $19,0 Y$ & rYq & 0 & $V, \varepsilon \varepsilon$ & $Y Y, \cdot \varepsilon$ & $r \cdot \lambda r$ & الثرقية & \multirow[t]{8}{*}{ برتقال طعم } \\
\hline$r$ & $1, V Y$ & $r \cdot, \leqslant 7$ & $1710 \leqslant$ & $r r, r \cdot$ & $T V V \leqslant V$ & $r$ & $9,1$. & $r, \wedge$. & $r \cdot \leq \wedge$ & المنوفية & \\
\hline$\varepsilon$ & 1,79 & 11,91 & $9 \leqslant 09$ & 15,07 & 10940 & $\varepsilon$ & $\lambda, 9 \Lambda$ & $1 Y, 79$ & IVVE & القليو بية & \\
\hline 7 & I, r & $1 \cdot, \cdot$, & VA9T & $\Lambda, \Lambda V$ & $1 . \leqslant r q$ & 7 & $\Lambda, Y \Lambda$ & 9 & 1109 & بني سويف & \\
\hline$r$ & I,VV & $V, r v$ & 0110 & $\lambda, \mathrm{VV}$ & 1.4 .9 & $r$ & $9, r^{2}$ & $V, 9 Y$ & 11.9 & البحيرة & \\
\hline V & 1,19 & 9,07 & Vo $\leqslant$ & $V, T r$ & A9V६ & $\mathrm{v}$ & 9,19 & 7,99 & 9VV & أسيوط & \\
\hline 1 & 1,90 & 0,10 & E.TV & $7, \vee \varepsilon$ & V१९q & 1 & $1 \cdot, r \varepsilon$ & $0, \leqslant 9$ & VTV & الغربية & \\
\hline- & $1, \leqslant 9$ & $1 \ldots$ & VA9 & $1 \ldots$ & $11 \times 00 \varepsilon$ & - & $\Lambda, \varepsilon)$ & $1 \ldots$ & 1 IraAr & جملة & \\
\hline$r$ & $1, r$, & $7 V, 04$ & $r r \leqslant 1 \wedge$ & $0 \xi, \lambda \Gamma$ & YAEYO & $r$ & $9, r 9$ & $\{q, r)$ & T.YV & أسيوط & \multirow[t]{5}{*}{ برتقال بذرة } \\
\hline$r$ & $1, \cdot \varepsilon$ & 11,00 & rAYq & $\mathrm{v}, \mathrm{v} \cdot$ & ए११६ & $r$ & $\Lambda, \cdot 0$ & $\Lambda, \cdot \Lambda$ & $\sum 97$ & سو هاج & \\
\hline 1 & 1,74 & $\Lambda, \uparrow \Lambda$ & $r \ldots v$ & $9,7 \pi$ & $\sum 99 \varepsilon$ & 1 & $1 ., 99$ & $V, \varepsilon \cdot$ & $\{0\}$ & الإسماعيلية & \\
\hline$\varepsilon$ & I,,$Y$ & $\varepsilon, 74$ & 1710 & $r, 1 \Lambda$ & $17 \leqslant \Lambda$ & $\varepsilon$ & 7,49 & $\varepsilon, Y \cdot$ & ron & المنيا & \\
\hline- & $1, \leqslant 9$ & $1 \ldots$ & $r \leqslant 77)$ & $1 \ldots$ & $01 \Lambda T A$ & - & $\Lambda, \Sigma \varepsilon$ & $1 \ldots$ & $71 r 9$ & جملة & \\
\hline
\end{tabular}

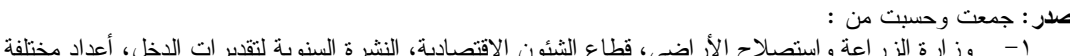

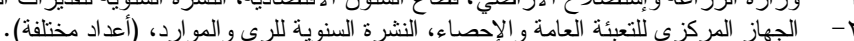


مما سبق يلاحظ أن المحافظات الغير رئيسية فى إنتاج البرتقال بسرة منل كفر الثيخ، الدقهلية، الإنها

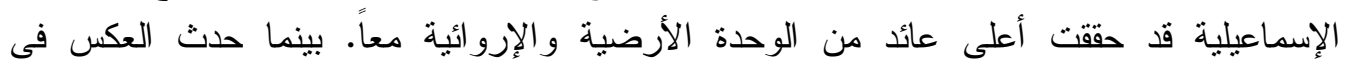

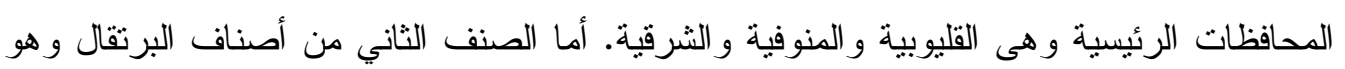
البرتقال الفالنشيا الصيفى فقد حققت محافظتى الإسماعيلية، البحيرة المركزين الأول و الثاني وفقاً

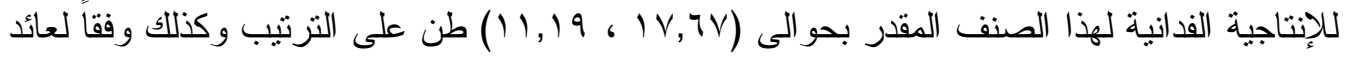

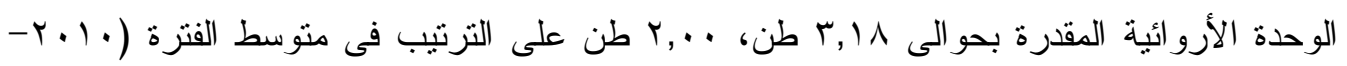

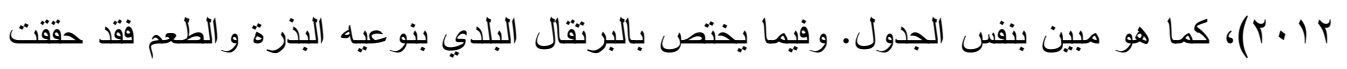

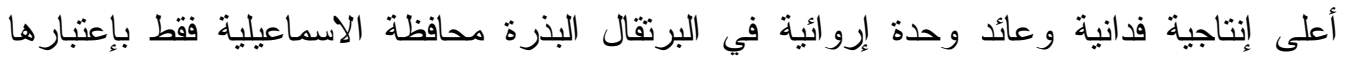

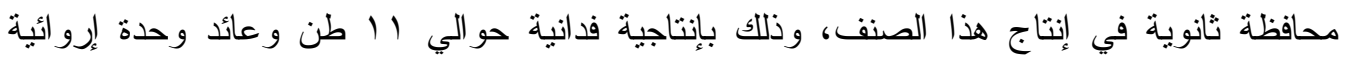

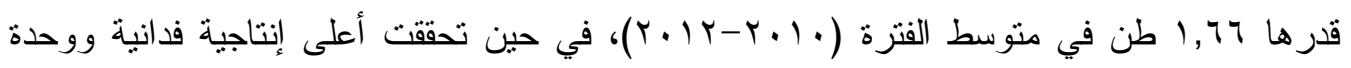

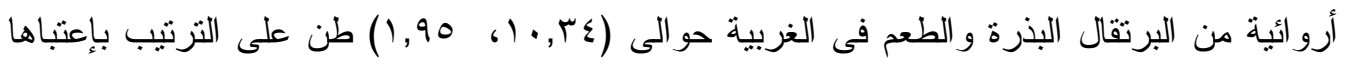

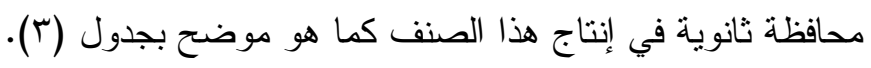

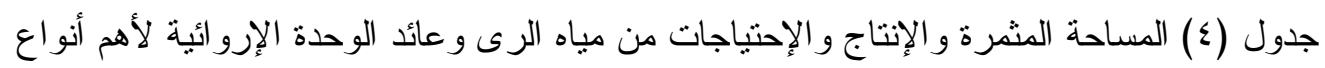

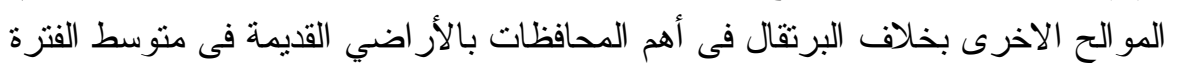

$(r \cdot \mid r-r \cdot 1 \cdot)$

\begin{tabular}{|c|c|c|c|c|c|c|c|c|c|c|c|}
\hline \multirow[t]{2}{*}{ الترتيب } & عائد الوحدة & \multirow[t]{2}{*}{$\%$} & من مياه الإحتيات & \multirow[t]{2}{*}{$\%$} & \multirow{2}{*}{ الإنتاج } & \multirow{2}{*}{ 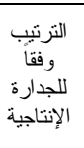 } & \multirow{2}{*}{ الفانتية } & \multirow[t]{2}{*}{$\%$} & المثرة & \multirow[t]{2}{*}{ المحافظات } & \multirow[t]{2}{*}{ الصنف } \\
\hline & (طن/... امץ & & (ألف مترب) & & & & & & (فدان) & & \\
\hline$\Lambda$ & $1, I T$ & $19, .1$ & rVqVA & $10, .0$ & $\leqslant Y \cdot \leqslant \leqslant$ & 9 & 0,91 & $r \cdot, 11$ & VIV & الثرقية & \multirow[t]{10}{*}{ يوسفى } \\
\hline$r$ & 1,7 & 9,10 & 19711 & $11, Y \wedge$ & r $10 . r$ & 7 & $\wedge, \S \wedge$ & $1 \cdot, 0$ & rVIT & البحيرة & \\
\hline V & $1,1 \mathrm{~V}$ & $M, \Sigma \leqslant$ & $r \leqslant \Lambda \leqslant V$ & $1 \cdot, \leqslant \Gamma$ & रqाई। & $r$ & $9, \cdot 7$ & $9, \cdot 9$ & TrIV & أسيوط & \\
\hline 0 & $1, \leqslant 1$ & T,VY & $1 \pi \leqslant 11$ & $7, \mathrm{VV}$ & $119 \cdot 1$ & V & $V, \Sigma\rceil$ & 8,17 & rors & المنوفية & \\
\hline 9 & $1, \cdot 1$ & $v, V I$ & 10499 & 0,94 & 17070 & $\Lambda$ & $7, V \varepsilon$ & 7,90 & $r \leq 09$ & المنيا & \\
\hline 7 & $1, r \wedge$ & $V, r$. & $1 \leqslant r \wedge 0$ & $V, \cdot 9$ & 19110 & $\varepsilon$ & $\Lambda, T Y$ & 7,0 & rYq9 & بني سويف & \\
\hline r & 1,19 & 0,7 & 11198 & $V, 09$ & YIY. & $T$ & $9, \leqslant Y$ & $7, Y 7$ & rYOI & الإنسماعيلية & \\
\hline$\xi$ & 1,09 & $\xi, \mathrm{V} \cdot$ & 9495 & $0, \pi 0$ & $1 \leqslant 9 \leqslant 0$ & 0 & $\Lambda, \S \Lambda$ & $9, \leqslant \lambda$ & TVTY & القليو بية & \\
\hline 1 & $T, \Sigma$, & $\cdot, 70$ & $1 \times 90$ & $T, I Y$ & rIr. & 1 & $T, 1 \varepsilon$ & $\cdot, V N$ & rov & كفر الثيخ & \\
\hline- & $1, r q$ & - & 199890 & $1 \cdots$ & TVqTY & - & $V, \wedge q$ & $1 \cdots$ & rorkV & جملة & \\
\hline$r$ & I,VY & rV,YT & \&ाTVY & $\varepsilon r, \wedge q$ & राII & T & $9, Y \leq$ & $r q, Y \leq$ & 1799 & الثرقية & \multirow{8}{*}{ لمالح } \\
\hline 7 &., 90 & $T \leqslant, 11$ & YTV. & $10, \mathrm{VT}$ & ro 199 & V & 0,91 & TI,VT & ETTY & الفيوم & \\
\hline 1 & 1,10 & $|\leqslant, T|$ & 17117 & $1 \Lambda, \leqslant 0$ & r9199 & $T$ & $9, \sqrt{7}$ & 10,71 & $r \cdot 7 r$ & البحيرة & \\
\hline$\xi$ & $1, \pi 0$ & $r, 01$ & YAOV & $r, \Gamma \wedge$ & глт. & 0 & $V, 10$ & Y,YO & $0 \leqslant$ & المنوفية & \\
\hline V & $\cdot, 90$ & $T, \leqslant V$ & TVTY & 1,7 & ro9A & $\varepsilon$ & $V, r \leq$ & I,A. & ros & أسيوط & \\
\hline 0 & 1,17 & $1, \Gamma \wedge$ & 10YV & $1,1$. & TVVA & 7 & 7,19 & $1, \leqslant 7$ & TAV & الإسماعيلية & \\
\hline$r$ & $1,7 r$ & $1, \Gamma \wedge$ & 10YA & $1,0 \leq$ & $r \leq q 1$ & $r$ & $\Lambda, V \leqslant$ & $1, \leqslant 0$ & rरo & القليوبية & \\
\hline- & $1, \leqslant 7$ & $1 \cdots$ & $11 . V A$. & $1 \cdots$ & 174.19 & - & $\Lambda, Y \uparrow$ & $1 \ldots$ & 19741 & جملة & \\
\hline 1 & $1, \wedge \Lambda$ & $\varepsilon V, 10$ & $V \xi$ & 00,01 & $1149 \varepsilon$ & 1 & $1 \cdot, 7 \leq$ & $\varepsilon \vee, T \leqslant$ & $1 \pi 1$ & البحيرة & \multirow{4}{*}{ ألياليا } \\
\hline$r$ & $1, Y 0$ & rr, . & $01 \xi$ & To,7ई & $7 \leqslant T$ & T & $V, \cdot V$ & rr,. 9 & 91 & الشرقية & \\
\hline$r$ & I, YT & TY,ru & 194 & $9, \xi 1$ & TMA & $r$ & $7,7 \leqslant$ & TY,rY & $r \leqslant$ & القليوبية & \\
\hline- & $1, T$ & $1 \cdots$ & 1004 & $1 \cdots$ & ro.1 & - & 9,11 & $1 \cdots$ & TVO & جملة & \\
\hline
\end{tabular}

جملة تتضمن محافظات أخرى

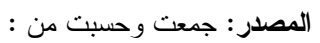
1- وز ارة الزر اعة وإستصلاح الأر اضي، قطاع الثئون الاقتصادية، النثرة السنوية لتنقير ات الدخل، أعداد مختلفة.

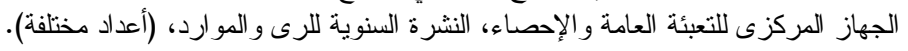

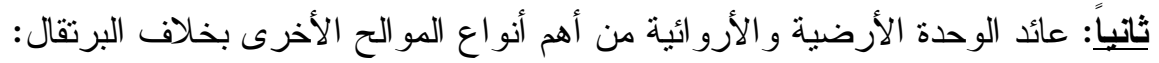

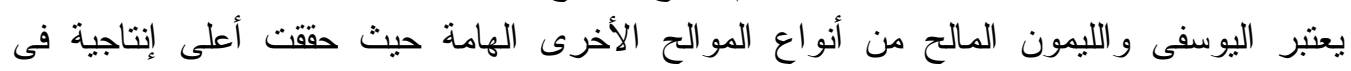

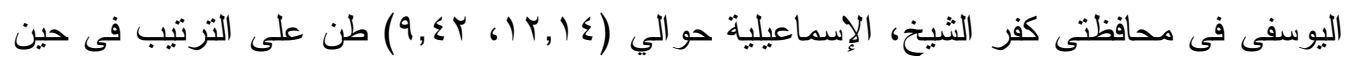

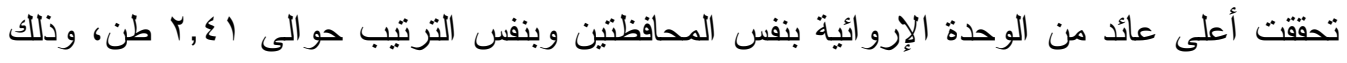

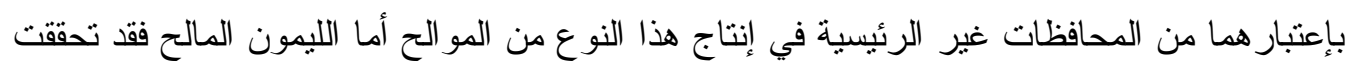

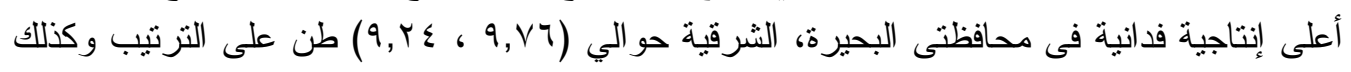




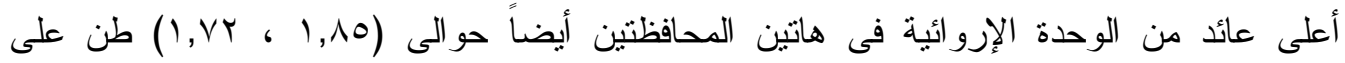

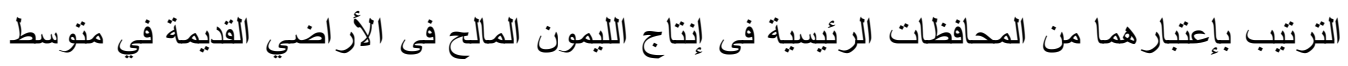

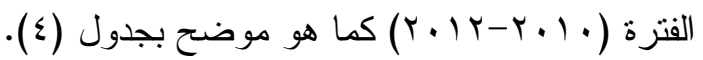

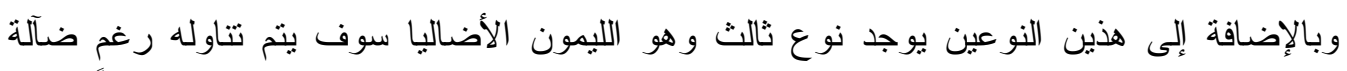

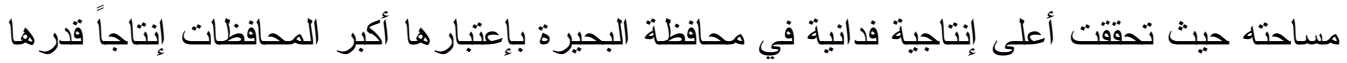

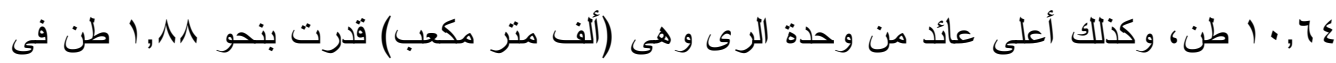

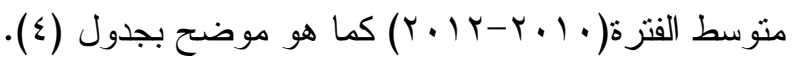

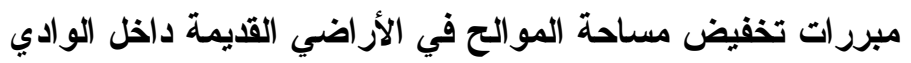

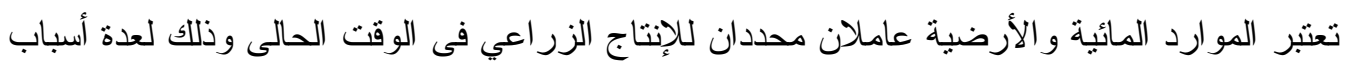

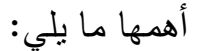

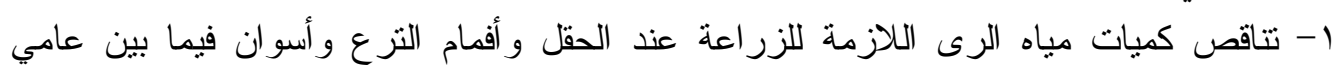

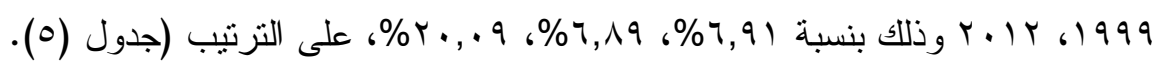

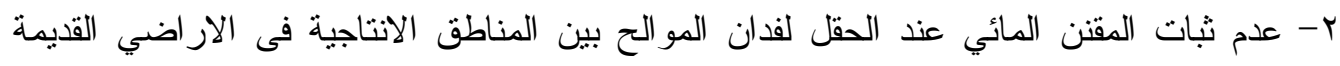

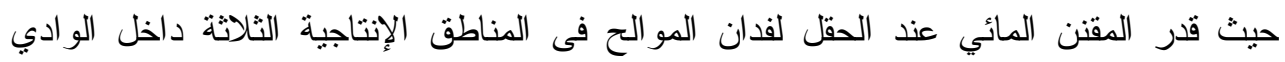

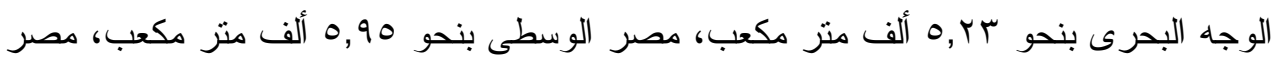

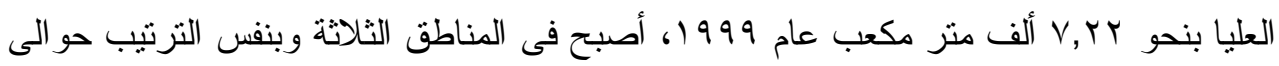

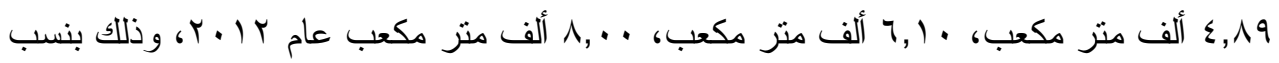

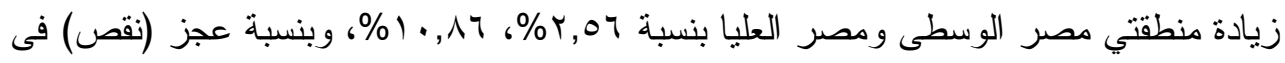

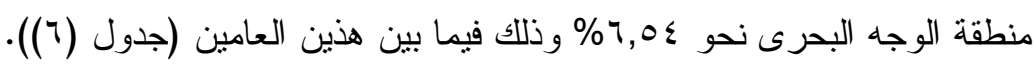

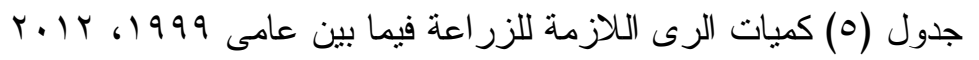

\begin{tabular}{|c|c|c|c|}
\hline \multicolumn{3}{|c|}{ كيات مياه الرى اللازمة للزر اعة (مليون متر مكعب) } & \multirow{2}{*}{ 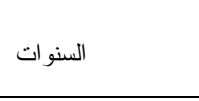 } \\
\hline عند أسو ان & عند أفمام الترع ع & عند الحقل & \\
\hline O.rrq & ห৭४тा & $r \leq \leqslant q r$ & 1999 \\
\hline$\varepsilon \cdot 1 r \Lambda$ & rา৭ห^ & Mr). 9 & r.IT \\
\hline$(1 \ldots 91)$ & $($ TVTr) & $(r \Uparrow \wedge \varepsilon)$ & مقدار النقص (التغير) \\
\hline$(r \cdot, \cdot 9)$ & $(\uparrow, \wedge ৭)$ & $(7,91)$ & 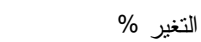 \\
\hline
\end{tabular}

القيم بين القوسين نمثل قيم سالبة

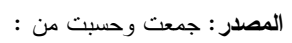

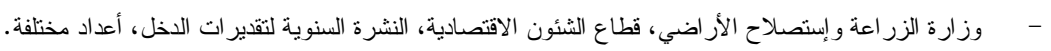
- - الجهاز المركزى للتعبئة العامة والإحصاء، النشرة السنوية للارى و المو ارد، (أعداد مختلفة).

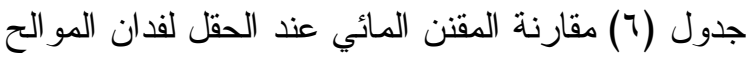

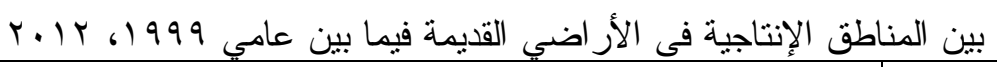

\begin{tabular}{|c|c|c|c|c|}
\hline \multicolumn{4}{|c|}{ المقنن المائي عند الحقل (متر مكعب/ فدان) } & \multirow[t]{2}{*}{ 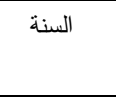 } \\
\hline متوسط عام & مصر العليا & مصر الوسطى & وجه بحرى & \\
\hline 00.9 & VTr. & $09 \leqslant 9$ & OrTr & 1999 \\
\hline 0011 & $\wedge \ldots \varepsilon$ & 71.1 & $\varepsilon \wedge 9$. & $r \cdot 1 r$ \\
\hline$r$ & $\vee \wedge \varepsilon$ & $10 \mathrm{r}$ & $(r \leqslant r)$ & 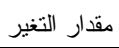 \\
\hline$\cdot, \cdot, \varepsilon$ & ד ד, 1. & r,07 & $(7,0 \leqslant)$ & التغير (\%) \\
\hline
\end{tabular}

- وزارة الزر اعة و إستصلاح الأراضي، قطاع الشئون الاقتصادية، النشرة السنوية لتقدير ات الدخل، أعداد مختلفة. الجهاز المركزى للتعبئة العامة و الإحصاء، النشرة السنوية للرى و المو ارد، (أعداد مختلفة). 


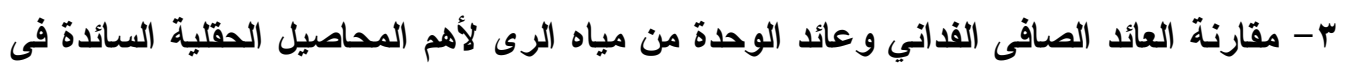

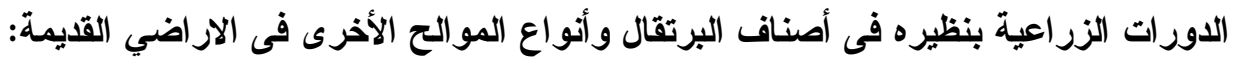

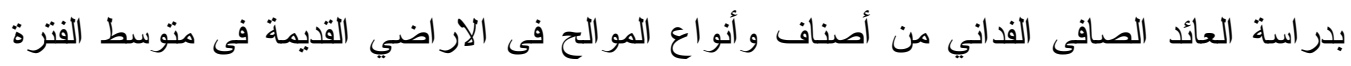

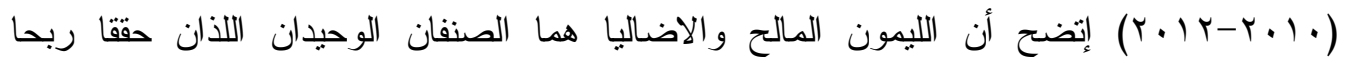

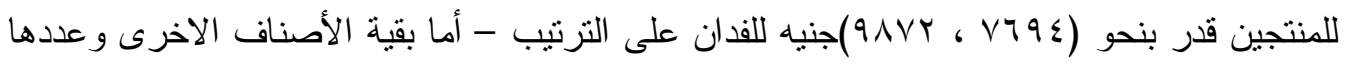

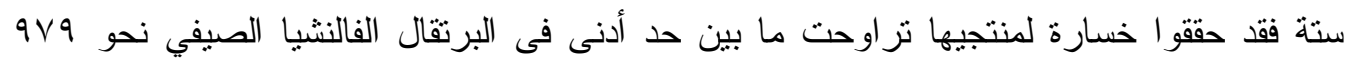

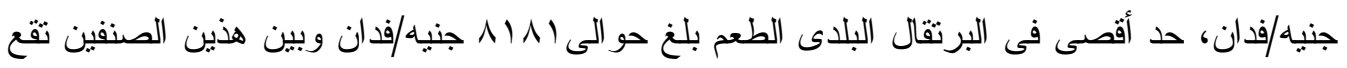

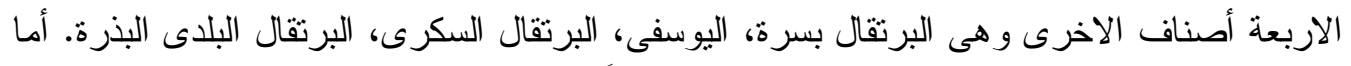

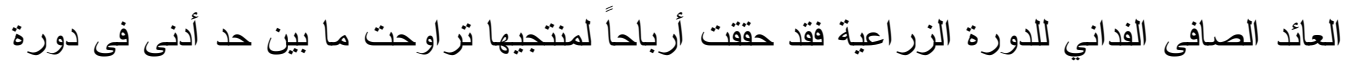

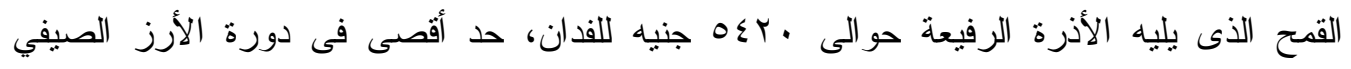

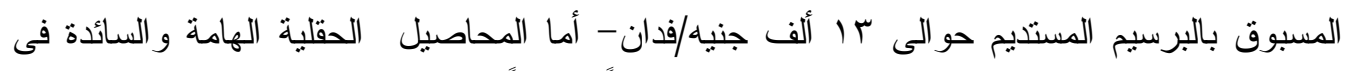

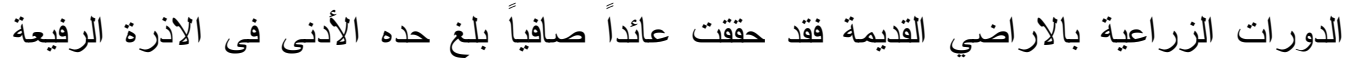

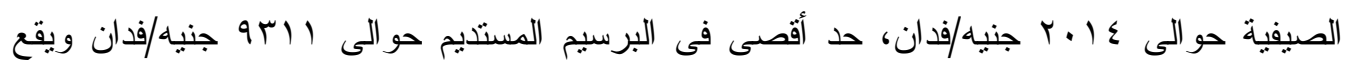

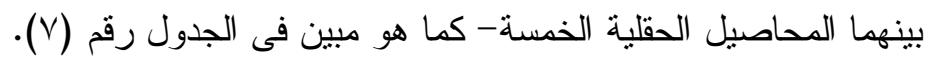

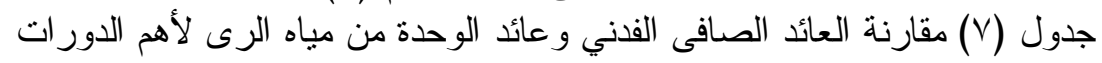

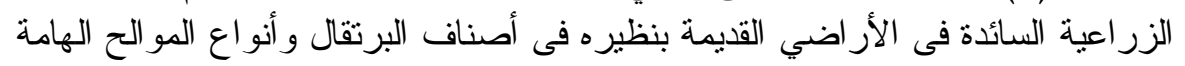

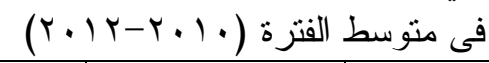

\begin{tabular}{|c|c|c|c|c|}
\hline عائد الوحدة من مياه & 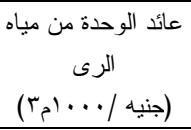 & 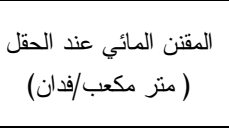 & (جنيد/فدان) الصافى & المو الح و أهم المحاصيل الزر اعية وأصناف \\
\hline$r$ & IVTV & $V \leqslant 77$ & IYq7V & برسيم مستديم+ أرز صيفي \\
\hline r & $r) \leqslant r$ & ד7rt & $M \cdot \Lambda$. & برسيم مستديم+ أذرة شامية \\
\hline$\varepsilon$ & 19r & 0101 & IITHO & برسيم مستديم+ أذرة رفيعة \\
\hline 1 & 1775 & $\leq \leqslant \leq 7$ & $v+q 1$ & برسيم تحريش + قطن \\
\hline 7 & 1.20 & TOVY & $V \cdot T r$ & قمح+ أرز صبفي \\
\hline 0 & $M \cdot r$ & $\leq V \leq r$ & $71 \times 0$ & قمح+ أذرة شامية \\
\hline $\mathrm{v}$ & 1.91 & $\leqslant 97 \leqslant$ & $0 \leqslant Y$. & قمح+ أذرة رفيعة \\
\hline$r$ & $(Y M V Y)$ & $07 \leq 7$ & $(9 \vee 9)$ & برثقال فالنشيا \\
\hline$\varepsilon$ & $(Y r \cdot r)$ & $07 \leqslant 7$ & $(171 \cdot)$ & برتقال بسرة \\
\hline$\circ$ & $(\operatorname{1\vee 71)}$ & $07 \leqslant 7$ & $(\Sigma \Gamma \wedge \Sigma)$ & يوسفى \\
\hline 7 & $(1,10)$ & $07 \leqslant 7$ & $(V 01 Y)$ & برتقال سكرى \\
\hline V & $(111 \cdot)$ & $07 \leqslant 7$ & $(\wedge) \varepsilon)$ & بلدى بذرة \\
\hline$\wedge$ & $(1.97)$ & $07 \leqslant 7$ & $(\wedge \backslash \wedge !)$ & بلدى طعم \\
\hline r & אדומו & $07 \leqslant 7$ & Vา9 & ليمون مالح \\
\hline 1 & $1 V \leq 1$ & $07 \leqslant 7$ & QAVT & ليمون أضاليا \\
\hline v & 719 & rroo & $r \cdot 1 \leq$ & أذرة رفيعة \\
\hline 0 & $91 \pi$ & r.r. & YV79 & أذرة شامية \\
\hline$\varepsilon$ & $\Delta T_{1}$ & rors & $r 9.7$ & قطن \\
\hline$r$ & 1994 & $18 \cdot 9$ & $r \leq \cdot T$ & قمح \\
\hline 1 & GYY & รᄉт & r7ot & أرز صيفي \\
\hline$r$ & $\varepsilon q r q$ & $9 \cdot 1$ & $\leqslant \leqslant 10$ & برسيم تحريش \\
\hline 7 & rove & rT.r & 9411 & برسيم مستديم \\
\hline
\end{tabular}


أما قيمة العائد من وحدة مياه الرى المقدرة بالألف متر مكعب فى الدورات السائدة فى الار اضي

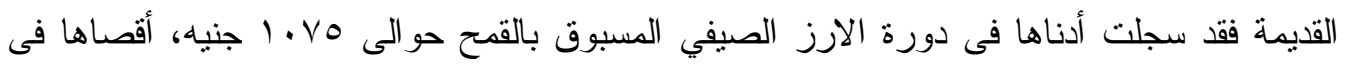
دورة الذرة الثامية المسبوق بالبرسيم المستديم حوالى بـ إب جنيه/فدان وذللك فى متوسط الفترة

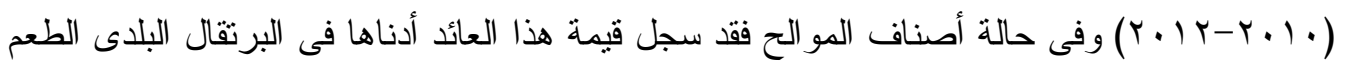

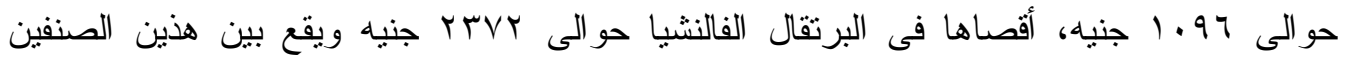
الأصناف الأخرى من الموالح - كما تراوح عائد الوحدة من مياه الرى فى الرئه المحاصيل الحقلية أدناه

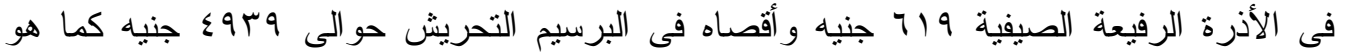

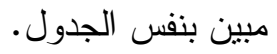
مما سبق يلاحظ أن المقنن المائي عند الحقل للفدان فى مختلف أصناف وأنواع الموالح قدر بنحو

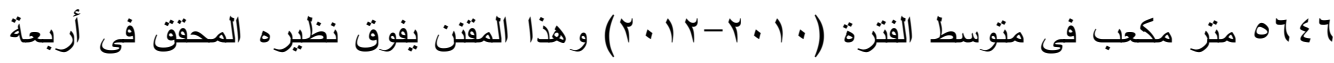

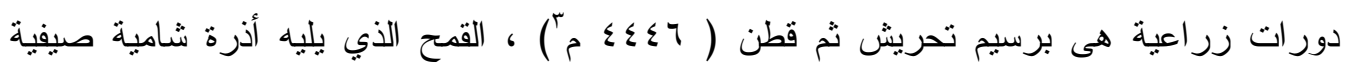

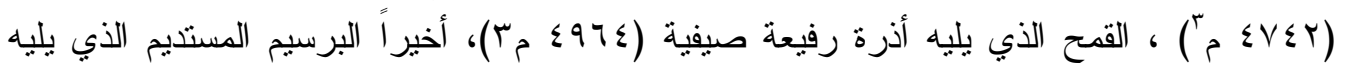

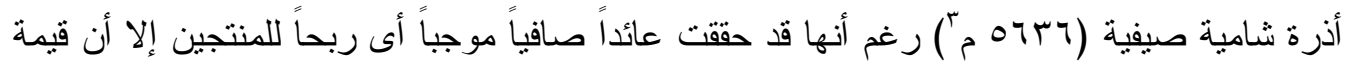

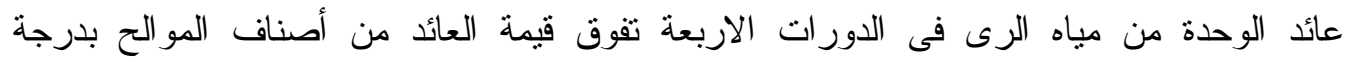
و واضحة. جدول (^) المؤشر ات الإقتصادية للمحاصيل الثتوية و الصيفية السائدة

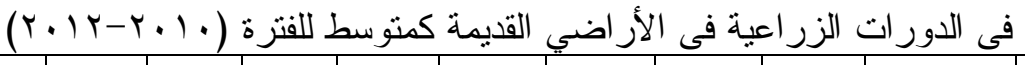

\begin{tabular}{|c|c|c|c|c|c|c|c|c|c|c|c|}
\hline بلدي & قصب & قطن & صنيفة & صيفي & شيفية أذرة & تحريش & بنجر & مستخيم & القمح & الوحدة & \\
\hline ar & rso & $\varepsilon \ldots$ & דזr & $\mid r \leqslant 1$ & IVVT & rq & ra7 & ror & ro.7 & ألف فدن & المسا.ة الكلية \\
\hline 1,rar & $\leq \wedge, \vee \cdot 1$ & $1, .79$ & r,YוA & $\{,, Y\}$ & $r, r q \leq$ & Ir,VOq & $r, T \leq 7$ & $r q, 1 r q$ & $r, Y \backslash A$ & طن/فدان & الإنتاجية \\
\hline 119 & $1 T \wedge \Lambda$. & $\leqslant r \wedge$ & VTr & $\leqslant 997$ & 0101 & rvol & $T \varepsilon \cdot V$ & rarq7 & 7111 & ألف طن & الإنتاج الكلى \\
\hline एव7ा & rro & $V \leqslant 1 \leqslant$ & $r \cdot \leqslant 1$ & $191 \mathrm{r}$ & $199 \mathrm{~V}$ & $\leqslant r_{0}$ & TYV & $\leqslant$ ro & rro. & جنية/طن & المزر عي \\
\hline$\leq v 1$ & $\leq 0.1$ & $\leqslant 0.1$ & $1 \leqslant V 7$ & $99 . r$ & $117 \lambda V$ & 174. & $r .97$ & 17911 & IOTY & مليون جنيه & قيمة الانتاج \\
\hline 0947 & lovr. & $10 \mathrm{VV}$. & $\leqslant V V Y$ & NIrv & Tגו & ONTO & vior & 11779 & $V \leqslant 7 \leqslant$ & جنيه /فدان & الإير اد الكلي \\
\hline$\leqslant .0 \leqslant$ & $V .1 V$ & $\mathrm{~V} .1 \mathrm{~V}$ & TVTY & $\leqslant \leqslant \wedge 1$ & $\varepsilon \cdot \leqslant \leqslant$ & iro. & rolv & rTON & $\varepsilon .01$ & جنية/فدان & التكاليف الكلية \\
\hline IANT & Avor & Avor & $r .1 \leq$ & r.07 & rviq & $\leqslant \leqslant 10$ & 4וा & 9411 & $r \varepsilon .7$ & جنيه/فدان & العائد الصافى \\
\hline$\cdot, \leq 7$ & 1, ro & 1, ro & $\cdot, \mathrm{VT}$ & - AT & 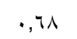 & r,Tr & 1,11 & $r, 90$ & $\cdot, \wedge \varepsilon$ & جنيه & أربحية الجنيه \\
\hline ITVY & $9 \wedge \wedge \wedge$ & $9 \wedge \Lambda \Lambda$ & rroo & 纟八tT & r.r & $9 \cdot 1$ & $r \cdot \lambda$. & rT.r & 18.9 & مَّ/فدان & المقنن المائى \\
\hline $1, .1 \mathrm{~V}$ & . & . & . & $\cdot, \lambda 广 \wedge$ & $1, \cdot 14$ & $1 \leqslant, .0 Y$ & $1 \cdot, \varepsilon \cdot V$ & 11,195 & $1,09$. & طن/... & عائد الوحدة \\
\hline$\varepsilon . r \wedge$ & $1 \pi q$. & $1 \pi q$. & 159. & $17 \leqslant 1$ & r) 199 & $411 \%$ & $r \varepsilon \cdot r$ & $\leqslant 179$ & rove & جنيه/... امّ & قيمة العائد \\
\hline
\end{tabular}

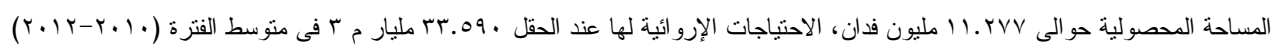
المصدر : جمعت وحسبت من: 1- وزارة الزر اعة و إسنصلاح الأراضي ، قطاع الثئون الإقتصادية، النشرة السنوية لتقيرات الدخل الزراعي، الإحصاءات الزر اعية، الجزء

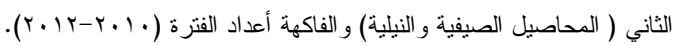

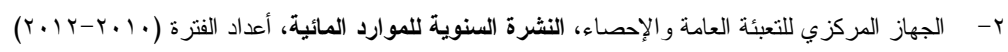


-الااتتاجية الفدانية لأهم المحاصيل الحقلية السائدة فى الدورات الزراعية بالأراضي القديمة و الار اضي الجديدة:

تثنير البيانات الواردة فى جدول رقم (9) الى إرتفاع الانتاجية الفدانية فى الار اضي القديمة لمحاصيل

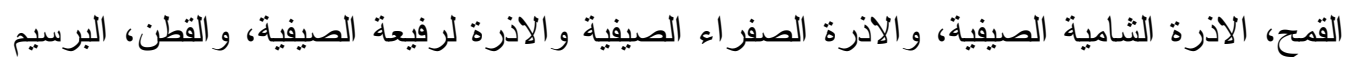

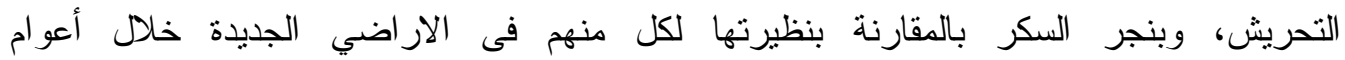

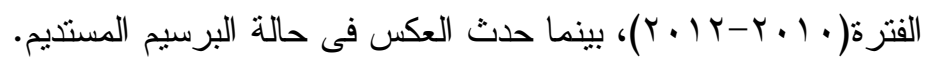
جدول (9) الإنتاجية الفدانية لأهم المحاصيل الحقلية السائدة فى الدورات الزر اعية فى الأراضي

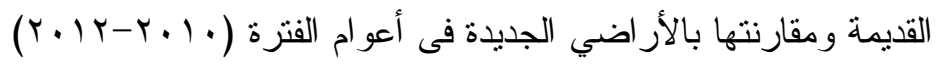

\begin{tabular}{|c|c|c|c|c|c|c|}
\hline ملاحظات & r.MT & $r .11$ & $r \cdot 1$. & المنطقة الانتاجية & الوحدة & المحصول \\
\hline \multirow{3}{*}{ التوسع فى زالار اضي اعته } & 19,11 & $1 \lambda, \vee \wedge$ & $17, \varepsilon Y$ & أر اضي قديمة (1) & \multirow{3}{*}{ أردب } & \multirow{3}{*}{ قمح } \\
\hline & $17, r Y$ & $17,1 \mathrm{~V}$ & $1 T, \pi T$ & أر اضي جديدة (؟) & & \\
\hline & $r, \wedge q$ & $r, 71$ & $r, \lambda$. & الفرق (Y-1) & & \\
\hline \multirow{3}{*}{ التوسع في زالار اضي اعته } & $r \varepsilon, r q$ & $r \varepsilon, r T$ & $Y Y, Y \varepsilon$ & أراضي قديمة (') & \multirow{3}{*}{ أردب } & \multirow{3}{*}{ أذرة شامية صيفية } \\
\hline & $r Y, T T$ & $r r, \cdot A$ & $r 1,79$ & أر اضي جديدة (؟) & & \\
\hline & $1,7 \pi$ & $1, r_{0}$ & $1, .0$ & الفرق (Y-1) & & \\
\hline \multirow{3}{*}{ التوسع في زالار اضي اعته } & YY,VY & $r Y, r)$ & $r 1, \cdot 1$ & أراضي قديمة ( (1) & \multirow{3}{*}{ أردب } & \multirow{3}{*}{ أذرة صفر اء صيفي } \\
\hline & $19,9$. & rI, Vo & $r,, 0$. & أر اضي جديدة (r) & & \\
\hline & $r, \wedge r$ &., 07 & $(\cdot, \varepsilon \Gamma)$ & الفرق (1-1) & & \\
\hline \multirow{3}{*}{ التوسع فيى زر اعته } & $17,1$. & 17,19 & 10,79 & أر اضي قديمة (') & \multirow{3}{*}{ أردب } & \multirow{3}{*}{ أذرة رفيعة صيفية } \\
\hline & ITVY & $|r, 0|$ & 11,10 & أر اضي جديدة (r) & & \\
\hline & $r, r \wedge$ & $r, \uparrow \wedge$ & $r, r \varepsilon$ & الفرق (Y-1) & & \\
\hline \multirow{3}{*}{ التوسع فى زالر اضي اعته } & 0,71 & $\mathrm{~V}, \mathrm{VO}_{\mathrm{O}}$ & 7,OY & أراضي قديمة (1) & \multirow{3}{*}{ قنطار } & \multirow{3}{*}{ قطن } \\
\hline & $0, \cdots$ & $V, 7 \lambda$ & $0, r$. & أر اضي جديدة (؟) & & \\
\hline & $\cdot, 71$ & $\cdot, \cdot V$ & $1, r Y$ & الفرق (1-1) & & \\
\hline \multirow{3}{*}{ التوسع فيى زالأر اضيه } & $1 Y, 7 \Lambda$ & IY,AY & IT,VT & أراضي قديمة (1) & \multirow{3}{*}{ طن } & \multirow{3}{*}{ برسيم تحريش } \\
\hline & 11,17 & $11, \varepsilon 1$ & $V, \varepsilon 0$ & أر اضي جديدة (r) & & \\
\hline & $1,0 Y$ & $1, \leqslant 1$ & $0, Y \wedge$ & الفرق (1-1) & & \\
\hline \multirow{3}{*}{ التوسع فى زالار اضي اعته } & $r q, 1$. & $r q, 1 r$ & $r 9,19$ & أراضي قديمة (1) & \multirow{3}{*}{ طن طن } & \multirow{3}{*}{ برسيم مستديم } \\
\hline & $r \cdot r \varepsilon$ & $r q, \varepsilon r$ & $r 9,01$ & أر اضي جديدة (؟) & & \\
\hline & $(1, r \varepsilon)$ & $(\cdot, r q)$ & $(0.39)$ & الفرق (Y-1) & & \\
\hline \multirow{3}{*}{ التوسع في زالار اضي اعته } & $r Y, 00$ & $r 1, r \varepsilon$ & $r, \cdot, r$ & أراضي قديمة (1) & \multirow{3}{*}{ طن } & \multirow{3}{*}{ بنجر السكر } \\
\hline & $19, r \mid$ & $\mid \lambda, \cdot 1$ & $I V, T$. & أر اضي جديدة (r) & & \\
\hline & $r, r \leq$ & rerr & $r, \varepsilon r$ & الفرق (Y-1) & & \\
\hline
\end{tabular}

المصدر : جمعت وحسبت من : (المبت ا- وزارة الزر اعة وإستصلاح الأراضي، قطاع الشئون الاقتصادية، نثرة الاقتصاد الزراعى، أعداد مختلفة.

\section{التكاليف الإنتاجية للموالح في الأراضي القديمة:}

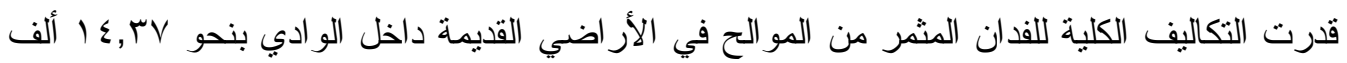

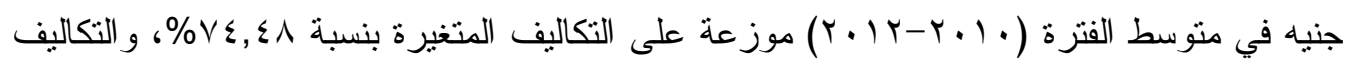

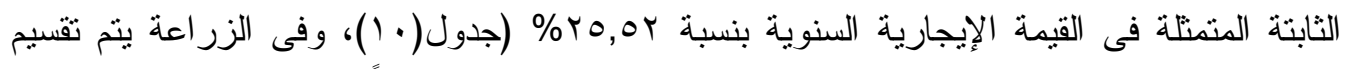

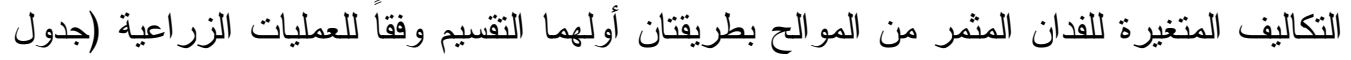

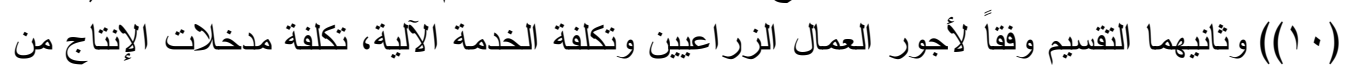

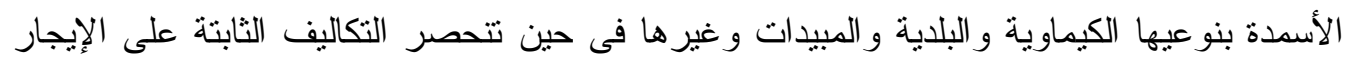

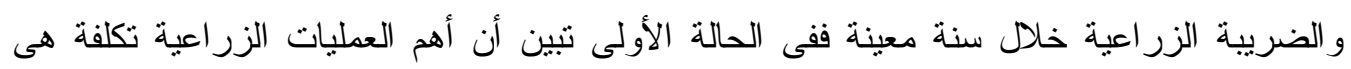

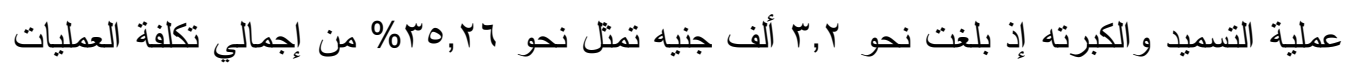
الزر اعية للفدان المثر من المو الح فى الأر اضي القديمة داخل الو ادي الدقدرة بحو الى ل, و ألف جنيه 


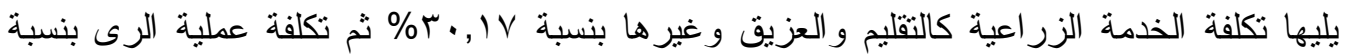
ل V V,VV

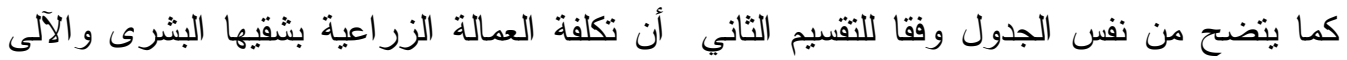

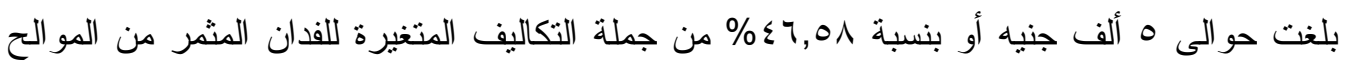

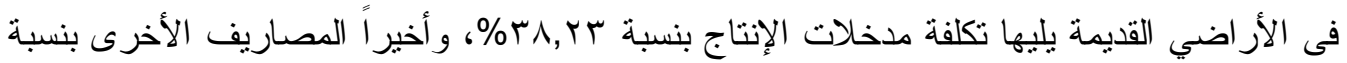
.\%10,19 هذا ويعتمد فى تأدية مختلف العمليات الزراعية على العمل البشرى بدرجة كبيرة حيث يمثل نحو ه^\%,rV الزراعية فى منوسط نفس الفترة، كما يحتاج الفدان المثر من المواله الح إلى أسمدة كيماوية قدرت

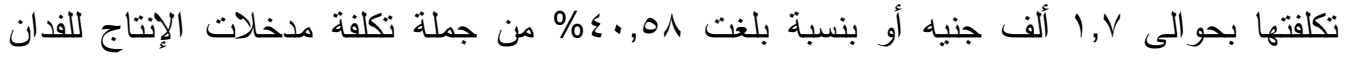

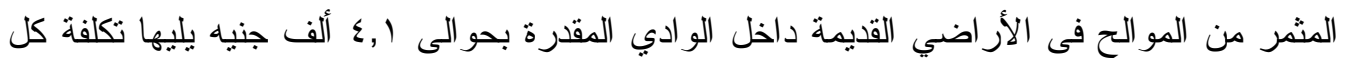

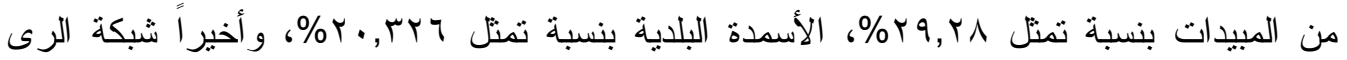

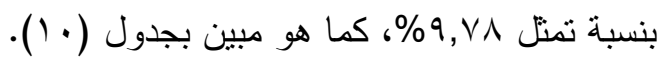
جدول (• (1) هيكل التكاليف الإنتاجية للفدان المثمر من الموالح في الأر اضي القديمة داخل الوادي

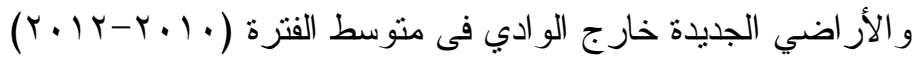

\begin{tabular}{|c|c|c|c|c|c|c|c|c|}
\hline \multicolumn{4}{|c|}{ الأر اضي الجديدة خارج الوادي } & \multicolumn{4}{|c|}{ الأر اضي القديمة داخل الو ادي } & \multirow{2}{*}{ بنود التكاليف } \\
\hline$\%$ & $\%$ & $\%$ & التكلفة(جنيه/فدان) & $\%$ & $\%$ & $\%$ & التكلفة(جنيه/فدان) & \\
\hline & & $r \Lambda, r V$ & $r \wedge \leqslant V$ & & & $r \cdot, I V$ & TVYA & الخدمة الزر اعبة \\
\hline & & $r \leqslant, 7 \varepsilon$ & $r \leqslant V T$ & & & $I V, V V$ & $171 \%$ & الرى \\
\hline & & $r q, \wedge 0$ & 1990 & & & $r 0, r_{T}$ & rr.. & التسميد و الكبرته \\
\hline & & $\mid V, I \varepsilon$ & IVY. & & & $17, \wedge$. & 10Y0 & مقاومة الآفات \\
\hline & $10,1 \mathrm{~V}$ & $1 \ldots$ & 1..ro & & $\Lambda \varepsilon, \wedge)$ & $1 \ldots$ & $q . \vee 4$ & جملة العطليات \\
\hline & & $\Lambda \varepsilon, Y \cdot$ & हА१Y & & & $\Lambda 9, r v$ & $\leqslant \leqslant 00$ & عمل بشرى \\
\hline & & $10, \lambda$. & $91 \wedge$ & & & $1 \cdot, \pi$ & or. & خدمة آلية \\
\hline$\varepsilon q, r)$ & & $1 \ldots$ & 011. & $\leq 7,01$ & & $1 \ldots$ & $\sum 910$ & جملة العمالة \\
\hline & & $\varepsilon r, r$ & IVAT & & & $\{\cdot, 0 \wedge$ & 177. & ثمن أسمدة \\
\hline & & $r V, \cdot r$ & $11 \leqslant r$ & & & rQ, ५A & 1191 & ثمن مبيدات \\
\hline & & IV,Vo & vo. & & & 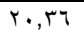 & הזr & ثئمن أسمدة بلدية \\
\hline & & $T r, \cdot Y$ & 00. & & & $9, \vee \wedge$ & $\varepsilon \cdots$ & شبكة رى \\
\hline To, T & & $1 .$. & EYro & $r \Lambda, r T$ & & $1 \ldots$ & 纟.91 & جملة المدخلات \\
\hline $1 \leqslant, \lambda T$ & $I \leqslant, \lambda T$ & & $I V \leqslant V$ & 10,19 & 10,19 & & 1747 & مصاريف أخرى \\
\hline $1 \ldots$ & $1 \ldots$ & $\vee \wedge, \varepsilon \vee$ & llvar & $1 \ldots$ & $1 \ldots$ & $V \varepsilon, \varepsilon \wedge$ & $1 \cdot v \cdot r$ & جملة التكاليف \\
\hline & & rI,or & TYTK & & & ro,or & T4TV & الإيجار السنوى \\
\hline & & $1 \ldots$ & 10.10 & & & $1 \ldots$ & $1 \leqslant 479$ & جملة التكاليف \\
\hline
\end{tabular}

النكاليف الكلية = التكاليف المتغيرة +الإيجار السنوى، المصاريف الأخرى مثل المصاريف الإدارية والإهلاك السنوى. التكاليف المتغيرة= تكلفة العمليات الزر اعية+ المصاريف الأخرى أو ( تكلفة العمالة الزر اعية+ تكلفة مدخلات الإنتاج+ الدصاريف الأخرى)

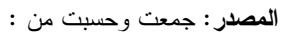
ب- وزارة الزر اعة و إستصلاح الأر اضي، قطاع الشئون الاقتصادية، النشرة السنوية لتقدير ات الدخل، أعداد مختلفة. r- الجهاز المركزى للتعبئة العامة والإحصاء، النشرة السنوية للرى و المو ارد، (أعداد مختلفة). مما سبق يلاحظ إرتفاع أثمان الأسمدة الكيماوية والبلدية وبالتالي إرتفاع تكلفة عملية التسميد نظر أ لإحتياجها إلى عمالة بشرية مكلفة . 


\section{الإير اد الكلي الصافى لأهم أنواع الموالح و أصناف البرتقال فى الأراضي القديمة:}

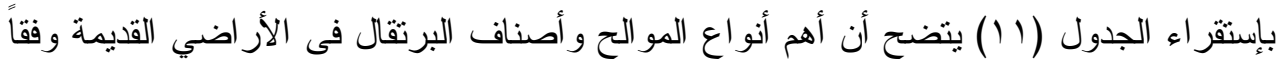

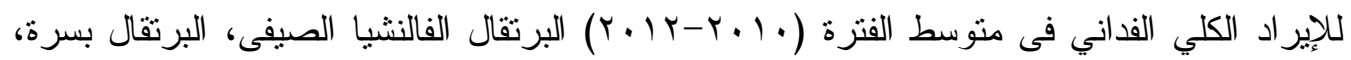

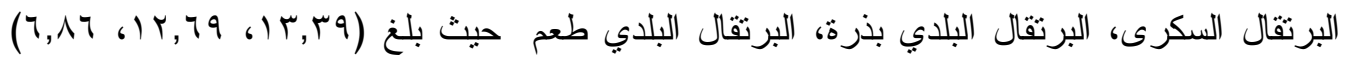

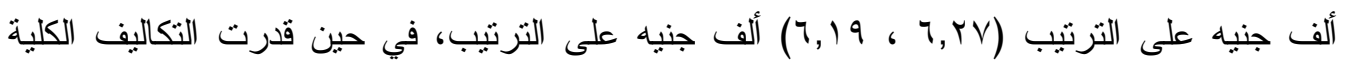

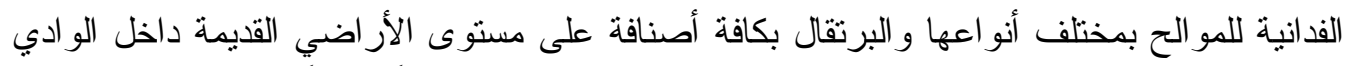

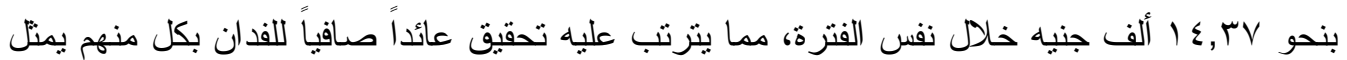

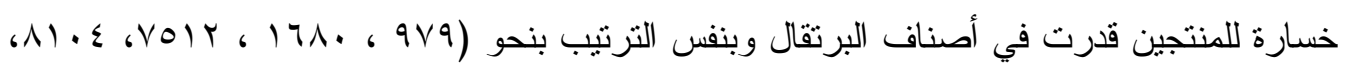

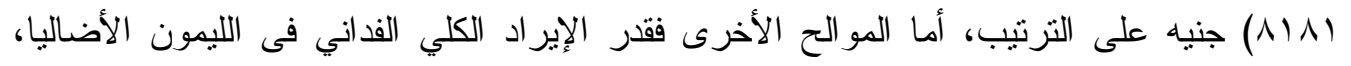

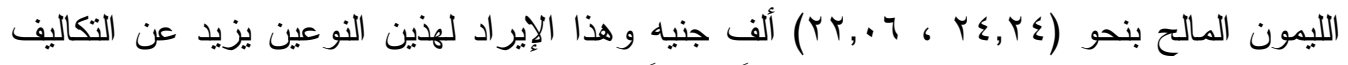

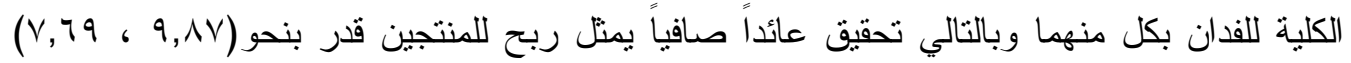

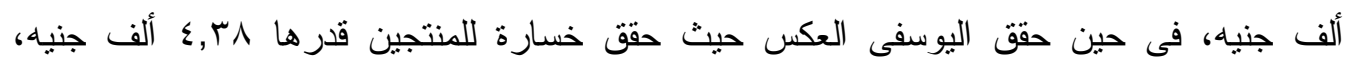

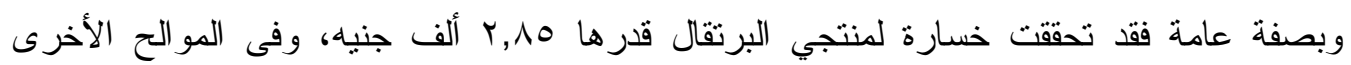

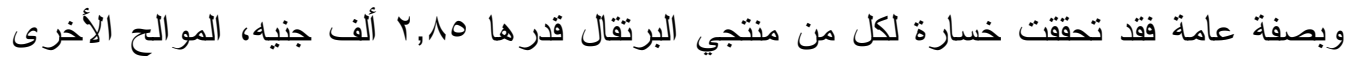

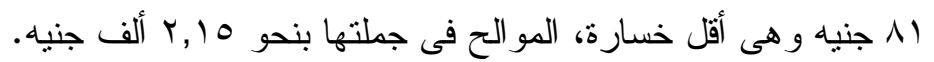

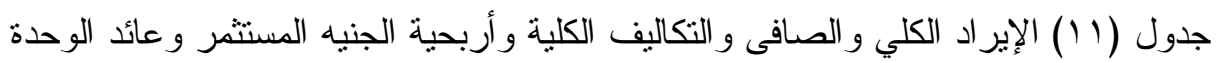

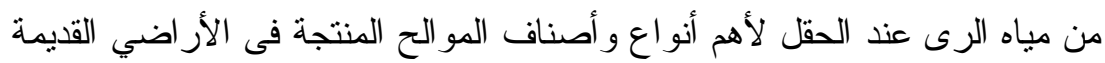

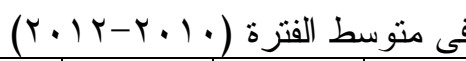

\begin{tabular}{|c|c|c|c|c|c|c|c|c|c|}
\hline \multicolumn{3}{|c|}{ عائد الوحدة الإرو ائية } & \multirow{2}{*}{ أربنية ألمنيهر } & \multirow{2}{*}{ 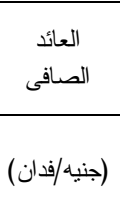 } & \multirow{2}{*}{ (الكلية } & \multirow{2}{*}{$\begin{array}{l}\text { إنتاج } \\
\text { (جنيه) } \\
\end{array}$} & \multirow{2}{*}{ المزرعي } & \multirow{2}{*}{ الالتتاجية } & \multirow{2}{*}{ و الأصناف الأف } \\
\hline والتًا لتِب & (جنيه) & كمنة & & & & & & & \\
\hline$\varepsilon$ & rtr.r & $1, V T$ & سالب & $(1 \uparrow \wedge \cdot)$ & $1 \leqslant r 79$ & $1 \% 7 \wedge 9$ & ITVE & 9,97 & برتقال بسرة \\
\hline$r$ & trut & 1,91 & سالب & $(9 \vee 9)$ & $1 \leqslant r 79$ & . & $1, x \leq 1$ & $1 \cdot, \vee \vee 9$ & فالنشيا \\
\hline 7 & 1410 & 1,Tr & سالب & $(V 0, Y)$ & $1 \leqslant r 79$ & TAOV & $v \leqslant \Lambda$ & $9,1 \vee$ & سكرى سقال \\
\hline$\Lambda$ & 1.97 & $1, \leqslant 9$ & سالب & $(\wedge \backslash \wedge)$ & $1 \leqslant r 79$ & $71 \wedge 1$ & VTr & $\Lambda, \leqslant 1$ & بلدى طعم \\
\hline v & 111. & 1,0 & سالب & $(\wedge) \cdot \varepsilon)$ & $1 \leqslant r 79$ & $7+70$ & $V \leqslant r$ & $\Lambda, \Sigma \varepsilon$ & بلدى بذرة \\
\hline & $r \cdot \varepsilon$. & $1, V Y$ & سالب & $($ ( $\wedge 01)$ & $1 \leqslant r 79$ & 11011 & 1119 & 9,79 & منتوسط \\
\hline 0 & 1871 & $1, \varepsilon$. & سالب & $(\varepsilon r \wedge \Sigma)$ & $1 \leqslant r 79$ & 9910 & $1 \times 70$ & $\vee, \wedge 9$ & يوسفى \\
\hline$r$ & $r 9.9$ & $1, \leqslant 7$ &., $0 \leqslant$ & Vา9 & $1 \leqslant r 49$ & TH.TH & YTVY & $\Lambda, Y_{\top}$ & ليمون مالح \\
\hline 1 & हYवा & יזד & $\cdot, 79$ & $9 \wedge \vee Y$ & $1 \leqslant r 79$ & $r \leq r \leq 1$ & rToג & $9,1 r$ & آليمون \\
\hline- & ror. & $1, \varepsilon r$ & & & $1 \leqslant r 49$ & $1 \leqslant Y \wedge \wedge$ & IVAT & $\Lambda, \cdot r$ & المتوسط الأخرى \\
\hline- & Y) TE & $1,7 \varepsilon$ & & & $1 \leqslant r 49$ & IYKIA & $1 \pi 19$ & $9, Y 7$ & منتوسط المو الح \\
\hline
\end{tabular}

القيم بين القوسين تمثل خسارة للمنتج أمام الصنف المناظر

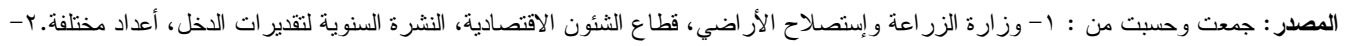

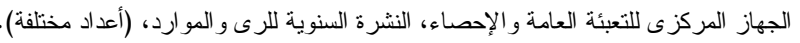

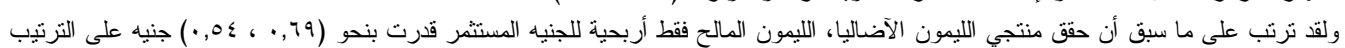
فى متوسط نفس الفترة ، كما هو مبين بنفس الجدول. 
العائد من الوحدة الإروائية لأهم أنواع الموالح وأصناف البرتقال في الأراضي القديمة:

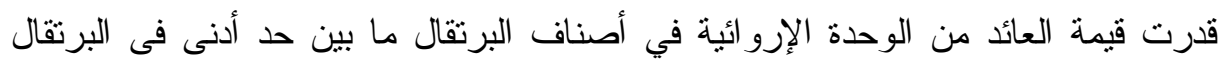

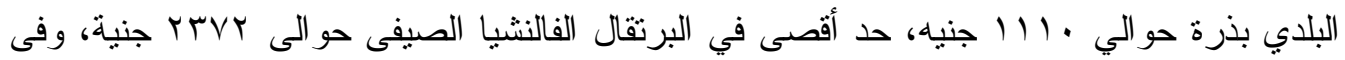

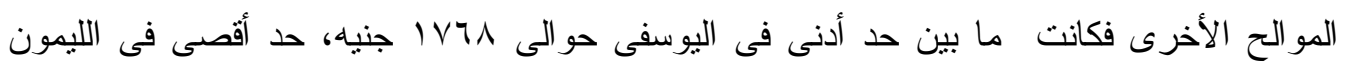

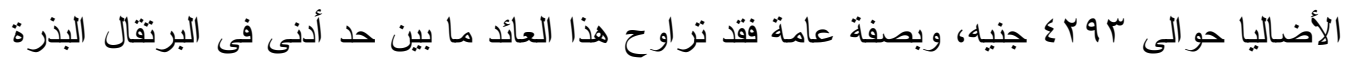

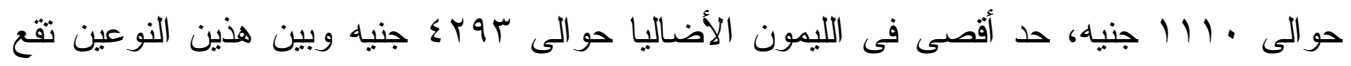

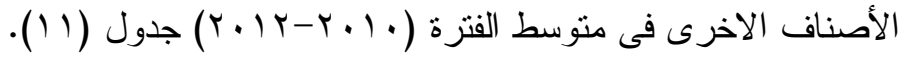

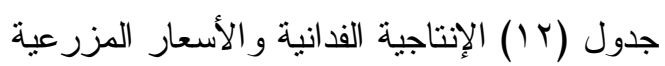

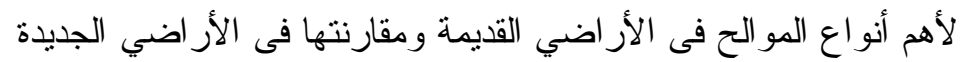

\begin{tabular}{|c|c|c|c|c|c|c|c|c|}
\hline \multirow[t]{2}{*}{ ملاحظات } & \multicolumn{3}{|c|}{ السعر المزرعي (جنيد/طن) } & \multicolumn{3}{|c|}{ الانتاجية الفدانية (طن) } & \multirow{2}{*}{ الإنتاجية } & \multirow{2}{*}{ الصنف أو } \\
\hline & T.IT & $r \cdot 11$ & $r \cdot 1$. & r.IT & $r \cdot 11$ & $r \cdot 1$. & & \\
\hline \multirow{5}{*}{ 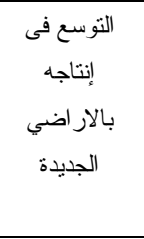 } & Irro & 1519 & $11 \%$. & $9,7 V$ & $9, \vee\rangle$ & $9,7 \wedge$ & أر اضي & \multirow{5}{*}{ أصناف } \\
\hline & & & & & & & قديمة & \\
\hline & ITTV & Irty & ITr. & $1 \cdot, 1 r$ & 9,97 & $1 \cdot, 01$ & أر اضي & \\
\hline & & & & & & & جديدة & \\
\hline & $r, \varepsilon r$ & r, १ & 1,9r & $\leq, 70$ & r,OV & $\Lambda, 0 \mathrm{~V}$ & التغير (\%) & \\
\hline \multirow{5}{*}{ زر الاعته فى من الحي } & KMA & IrVo & IrTI & $\wedge, 1 \wedge$ & $v, q$. & $v, 7)$ & أر اضي & \multirow{5}{*}{ يوسفى } \\
\hline & & & & & & & قديمة & \\
\hline & $1 r \ldots$ & 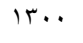 & IrV. & 9,19 & $9, .0$ & $\wedge, \wedge 9$ & أر اضي & \\
\hline & & & & & & & جديدة & \\
\hline & r & 1,97 & $r, I V$ & Ir, ro & $1\{, 07$ & $17, \lambda r$ & التغير (\%) & \\
\hline \multirow{5}{*}{ زر العته فى من الار اضي } & $r v \ldots$ & rצAT & THYA & ᄉ, Or & 1,17 & $\Lambda, M$ & أر اضي & \multirow{5}{*}{ الليالح } \\
\hline & & & & & & & قديمة & \\
\hline & TVY. & r.q. & r४10 & 9,90 & 9,91 & $9, \Upsilon^{\top}$ & أر اضي & \\
\hline & & & & & & & جديدة & \\
\hline & $\cdot, \vee \leqslant$ & $\cdot, 10$ & r.IV & $17, \mathrm{VA}$ & YI,rv & $1 \varepsilon, \cdot \varepsilon$ & التغير (\%) & \\
\hline \multirow{8}{*}{ 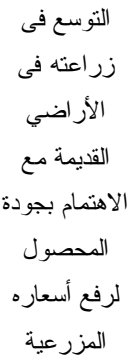 } & rTVt & r770 & rutq & $1 \cdot, r \cdot$ & $1 \cdot, 1 \varepsilon$ & $V, \Sigma V$ & أر اضي & \multirow{8}{*}{ الأضاليا } \\
\hline & & & & & & & قديمة & \\
\hline & หr99 & rדצV & rTAY & $\varepsilon, 79$ & r,Tr & r, Yo & أر اضي & \\
\hline & & & & & & & جديدة & \\
\hline & $\cdot, 9 \mathrm{~V}$ & $\cdot, \cdot \wedge$ & 1, ז & $(0 \varepsilon, \cdot r)$ & $(V \varepsilon, \cdot T)$ & $(7 r, 19)$ & التغير (\%) & \\
\hline & & & & & & & & \\
\hline & & & & & & & & \\
\hline & & & & & & & & \\
\hline
\end{tabular}

المصدر : جمعت وحسبت من :

ا - وز ارة الزر اعة و إستصلاح الأر اضي، قطاع الشئون الاقتصادية، النشرة السنوية لتقدير ات الدخل، أعداد مختلفة.

ץ- الجهاز المركزى للتعبئة العامة والإحصاء، النشرة السنوية للرى و المو ارد، (أعداد مختلفة).

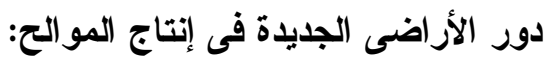

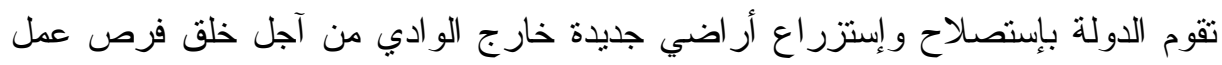

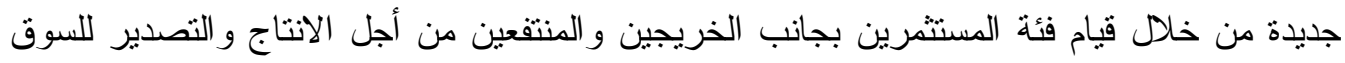

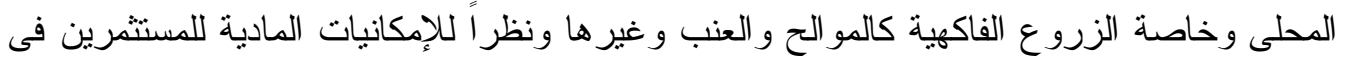

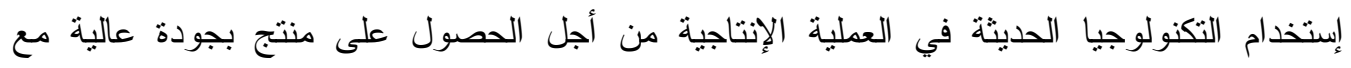




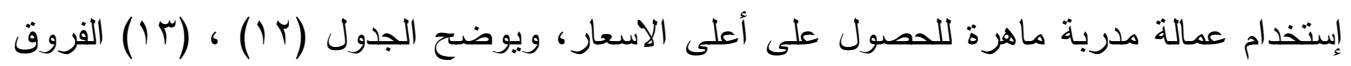

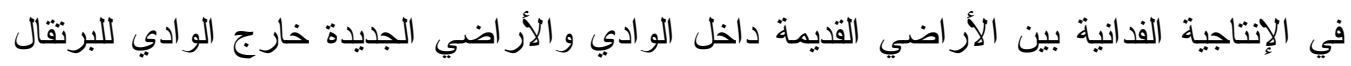

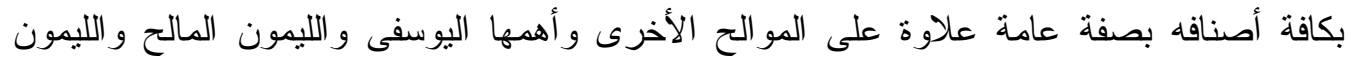

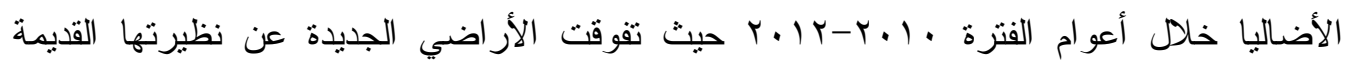

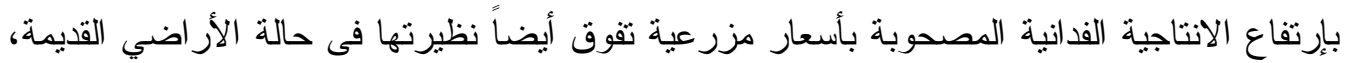
وبالتالي تخفيض تكلفة الوحدة المنتجة وحصول المنتجين على دخول صافية صافية مجزية.

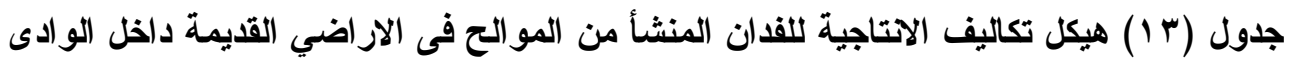

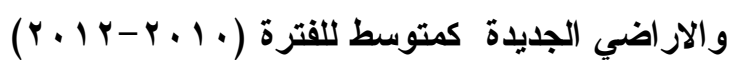

\begin{tabular}{|c|c|c|c|c|c|c|c|c|}
\hline \multicolumn{4}{|c|}{ أر اضي جديدة خار ج الو ادي } & \multicolumn{4}{|c|}{ أر اضي قديمة داخل الو ادي } & \multirow[b]{2}{*}{ بنود التكاليف } \\
\hline & $(\%)$ & $(\%)$ & 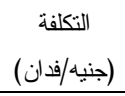 & & $(\%)$ & $(\%)$ & 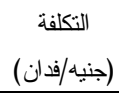 & \\
\hline & & IV,YY & ros & & & 10,1 . & 191. & تحضير الأرض وزر اعة \\
\hline & & 10,91 & rrot & & & $r \cdot, r$ & roth & الخدمة الزر اعية \\
\hline & & $r V, \cdot r$ & $0 \leqslant 0$. & & & $r \varepsilon, \mu r$ & $\varepsilon r \leqslant 0$ & الرى \\
\hline & & $r u, r$. & TIKr & & & rY,OV & r100 & التشميد \\
\hline & & $\Lambda, 0 \varepsilon$ & IrOV & & & 8,74 & 979 & مكافحة الافات \\
\hline & $\Delta T, \& 1$ & $1 \ldots$ & $1 \leqslant V Y \leqslant$ & & ᄉ & $1 \ldots$ & IrTEV & جملة العمليات الزر اعية \\
\hline & & $v 9, .1$ & $0 . \leqslant 0$ & & & $\Delta \vee, v \varepsilon$ & $\varepsilon \wedge 0$. & عمل بشرى \\
\hline & & $r \cdot, Q Y$ & irro & & & IT,YT & TVA & عمل آلي \\
\hline \multirow[t]{6}{*}{$r v, \Sigma \varepsilon$} & & $1 \ldots$ & TrA. & $r V, \lambda r$ & & $1 \ldots$ & OOYA & جملة العمالة الزر اعية \\
\hline & & 10,77 & $\mu \cdot v$ & & & Ir,VV & $9 \wedge$. & ثمن شتلات \\
\hline & & $1 \wedge, 9 \varepsilon$ & 101. & & & $i v, \lambda$. & IYTV & ثمن أسمدة كيماوية \\
\hline & & $11, \mathrm{rA}$ & qAT & & & $1 r, 11$ & ATV & ثمن أسمدة بلدية \\
\hline & & $\mathrm{v}, 7 \wedge$ & $7 \leqslant 1$ & & & $\wedge, 0$. & 7.0 & ثمن مبيدات \\
\hline & & $\varepsilon 0,9 \varepsilon$ & rarr & & & $\varepsilon \vee, V \uparrow$ & $r \varepsilon \ldots$ & شبكة رى \\
\hline$\varepsilon \wedge, q \vee$ & & $1 \ldots$ & $\Delta \pi \leq \varepsilon$ & $\varepsilon \wedge, V)$ & & $1 \ldots$ & 2119 & جملة الدذخلات الإنتاجية \\
\hline 15,09 & $1 r, 09$ & & rMIT & $1 r, \xi V$ & $1 T, \varepsilon V$ & & 1971 & مصاريف أخرى \\
\hline \multirow[t]{3}{*}{$1 \ldots$} & $1 \ldots$ & $10, \cdot r$ & IV. & $1 \ldots$ & $1 \ldots$ & Ar, QV & $1 \leq 710$ & جملة التكاليف المتغيرة \\
\hline & & $1 \varepsilon, 9 V$ & $r \ldots$ & & & $V, \cdot r$ & $r \ldots$ & الإيجار السنوى \\
\hline & & $1 \ldots$ & r... & & & $1 \ldots$ & |VT10 & جملة التكاليف الكلية \\
\hline
\end{tabular}

المصاريف الأخرى مثل المصاريف الإدارية والإهلالك السنوى وتكلفة مصادات الرياح مثل زر اعة أشجار الجازورينا المصدر : جمعت وحسبت من : المصني ا- - وز ارة الزر اعة و إستصلاح الأر اضي، قطاع الشئون الاقتصادية، النشرة السنوية لنقدير ات الدخل، أعداد مختلفة.

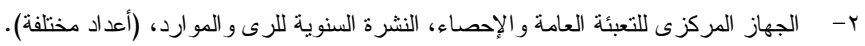

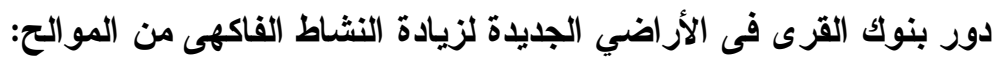

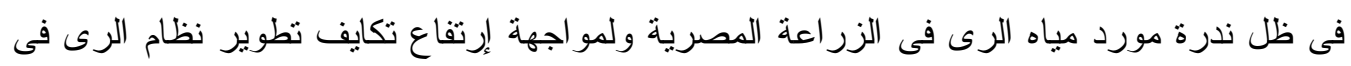

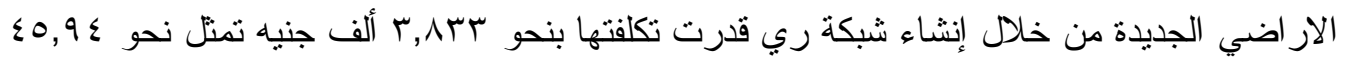

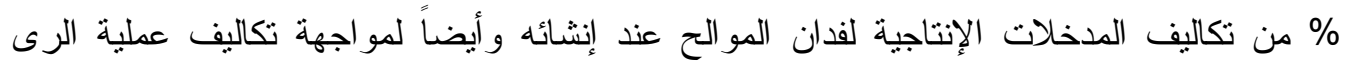

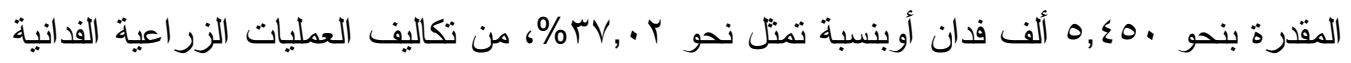

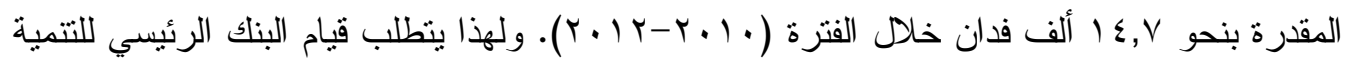

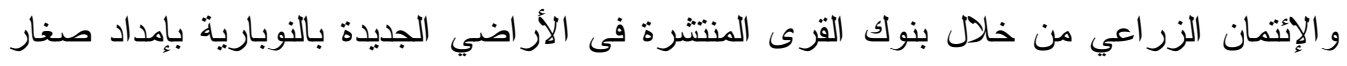

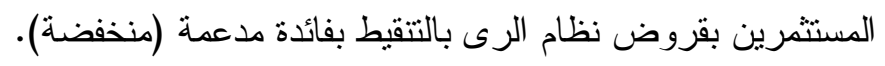




\section{الملخص والتوصيات}

تنين من النتائج البحثية أن الموالح في الأراضي القديمة تحتل المركز الأول بين انواع المات

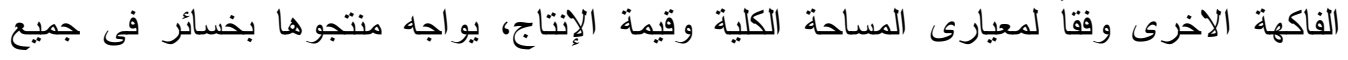

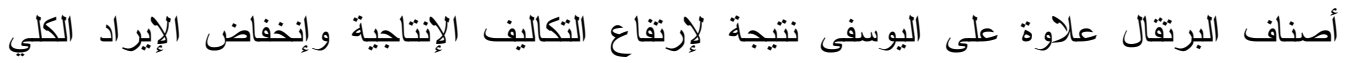

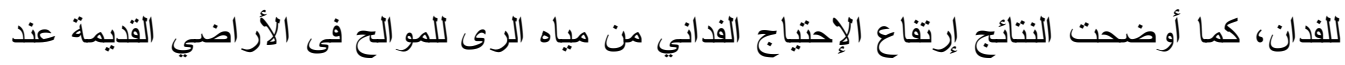

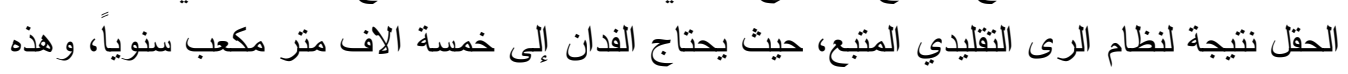

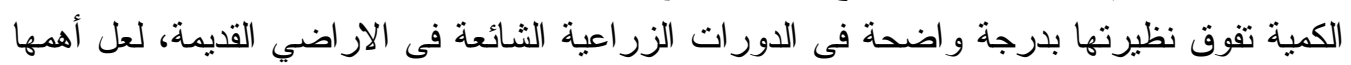

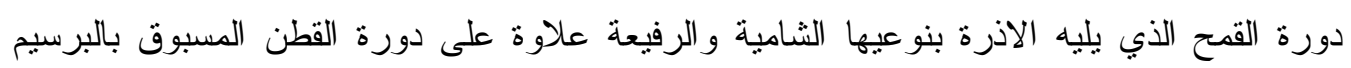

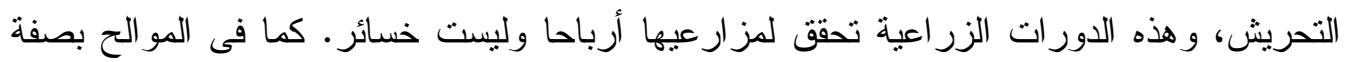
عامة، ولقد ترتب على إرتفاع الاحتياج الفدانى من مياه الرى للمو الح إنخفاض عاضئ عائد الوحدة الارو ائية

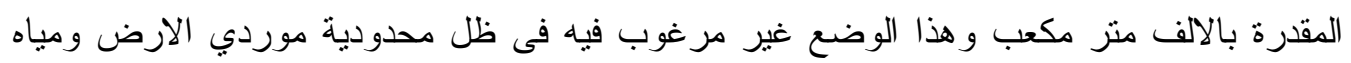

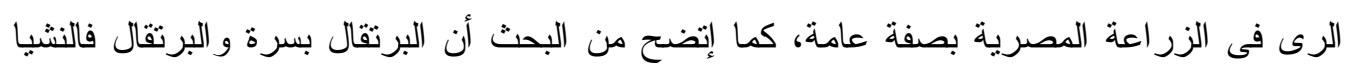
من أهم أصناف البرتقال في الأر اضي القديمة.

كما تبين من البحث أن أعلى إنتاجية من الوحدة الأرضية ( الفدان)، وأقصى عائد للوحدة

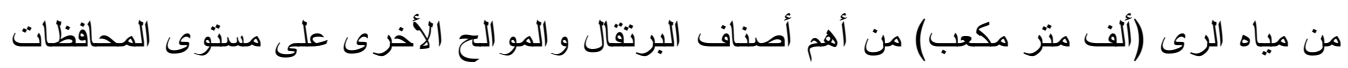

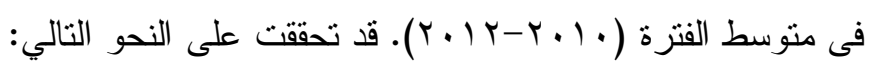
- البرتقال بسرة أربع محافظات: (كفر الثيخ، الدقهلية ، البحيرة ، الإسماعيلية)-البرنقال الفئ الفالنشيا

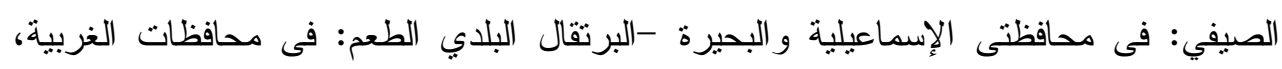

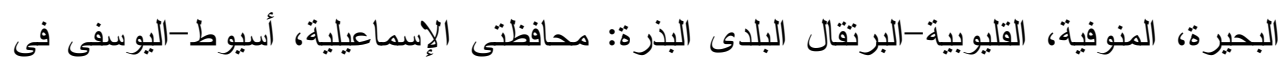

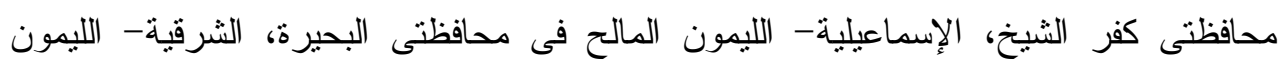
الأضاليا فى محافظتى الشرقية و البحيرة. - كما اتضح أن التكاليف الإنتاجية الكلية للفدان المثر من الموالح فئى الأر اضي القديمة فى متوسط

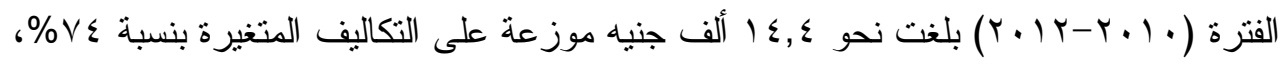

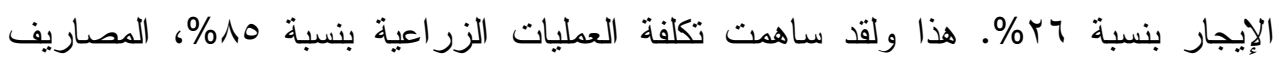

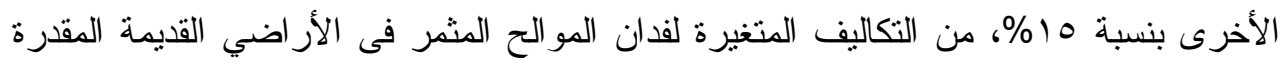

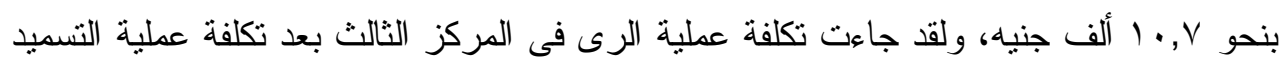

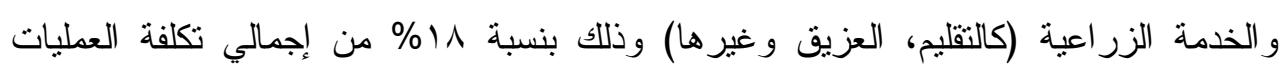

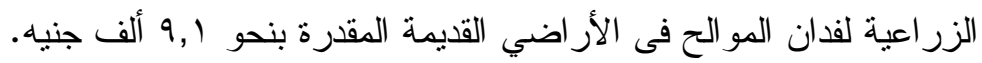

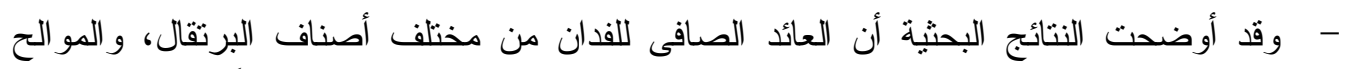

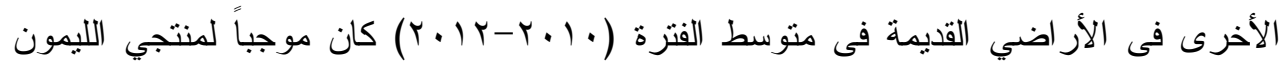

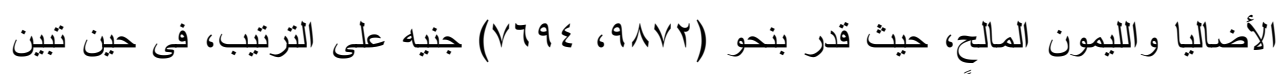

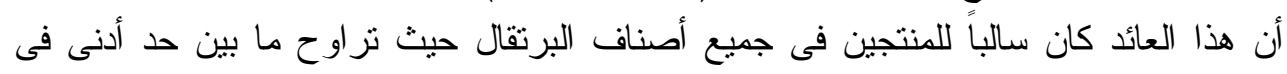

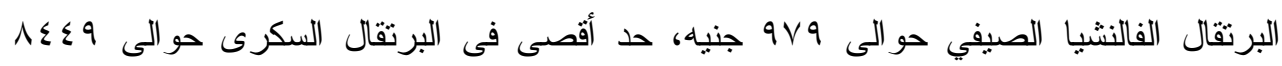

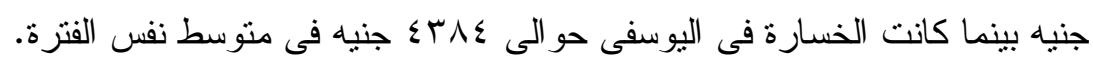

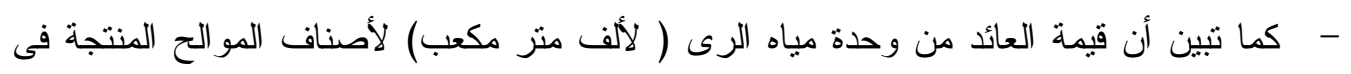

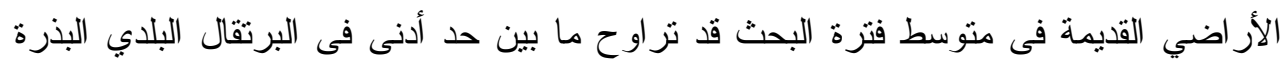


حوالى 111 جنيه، حد أقصى فى الليمون الآضاليا حو الى بوج؟؛ جنيه وبين هاتين القيمنين تقع

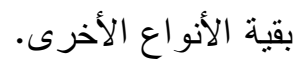

\section{التوصيات}

( ) إحلال زر اعة المحاصيل التقليدية الحقلية الاستر اتيجية بدلا من زر اعة الأصناف من الموالح

التى تحقق خسارة.

r) التوسع فى إستخدام العمل الآلى لإسهامه فى خفض خلى التكاليف الإنتاجية الكلية الفدانية

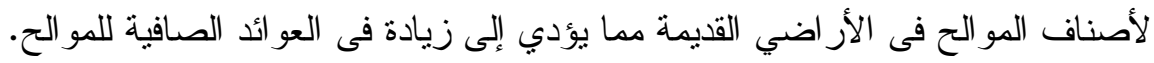

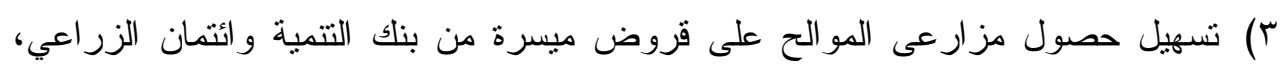
لإزتفاع تكاليف إنشاء الفدان.

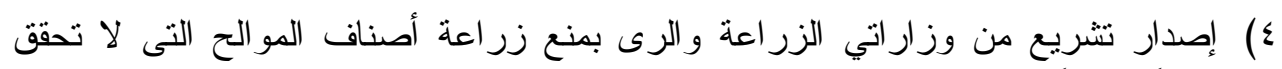

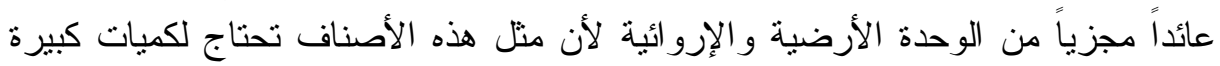

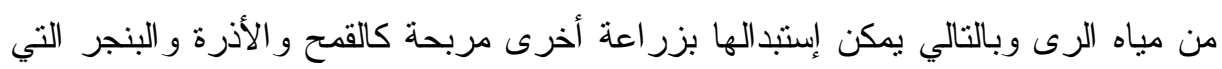

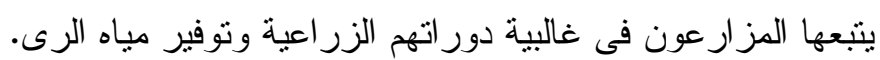

\section{المر اجع}

(1) عبدالحليم عبد النبى عبدالحليم، دراسة إقتصادية لإنتاج وتصدير الموالح المصرية، رسالة

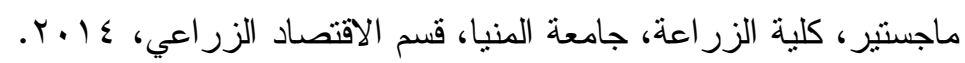

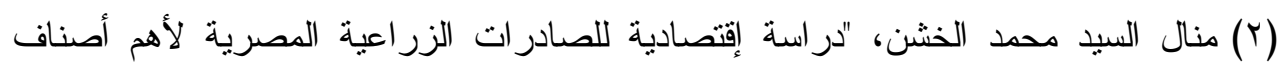

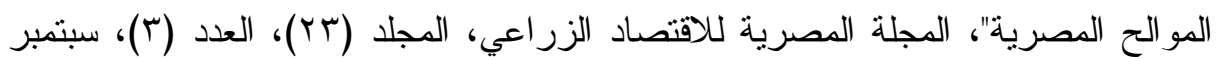

(r) ناصر محمد عوض تمام، "محددات الطلب العالمى على بعض الصادرات البستانية

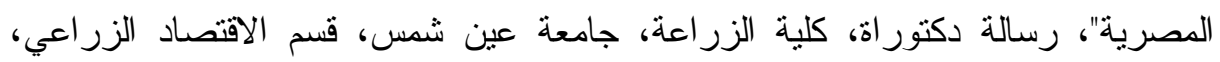

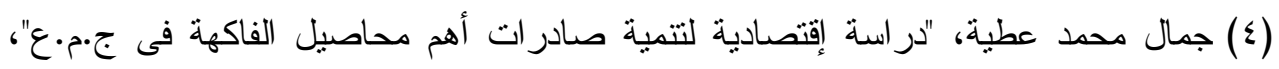

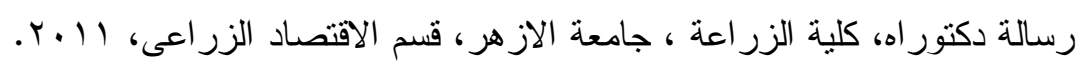

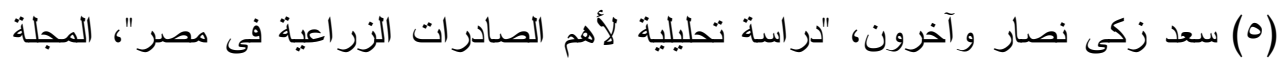

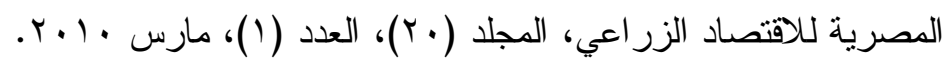

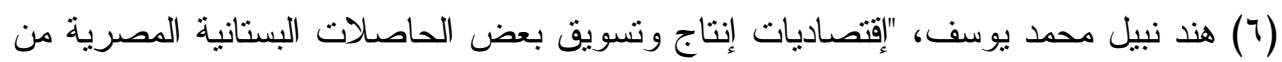

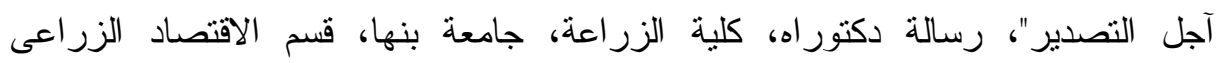

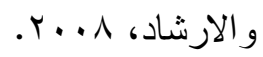

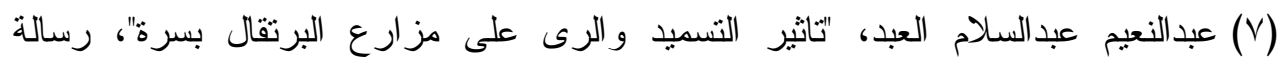

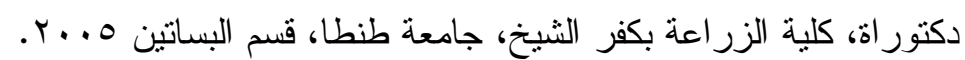

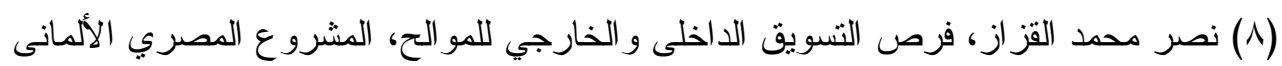

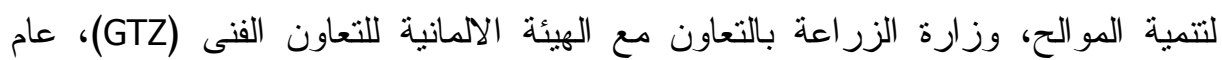
. Y...O

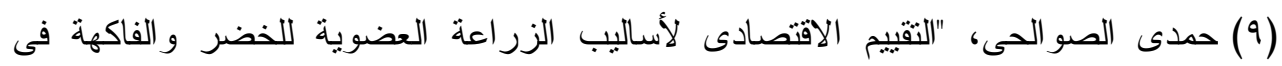

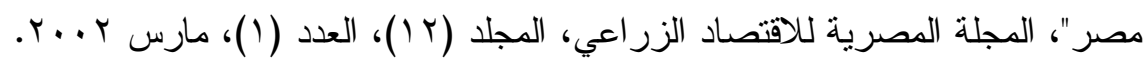




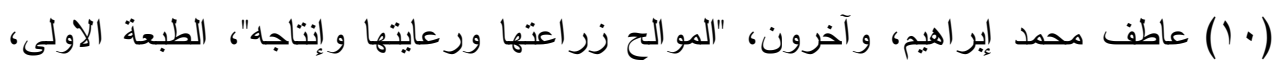

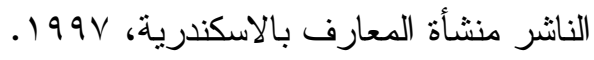

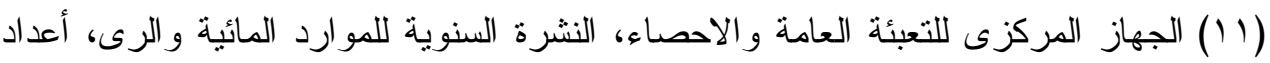

مختلفة.

(r ( ) وزارة الزراعة وإستصلاح الأراضي، قطاع الثشئون الإقتصادية، النشرة السنوية لتقدير

الدخل الزر اعي، (أعداد متفرقة).

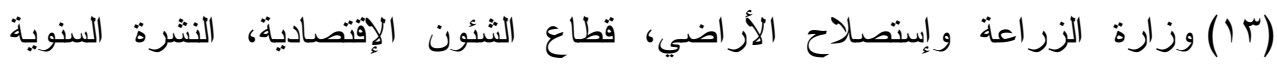

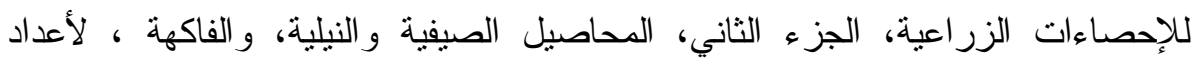

مختلفة.

(ع () وزارة الزر اعة وإستصلاح الأر اضي ، قطاع الشئون الإقتصادية، إحصاءات الأسعار

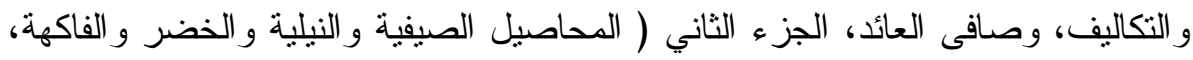

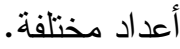

(15) Richard H.Leftwich, The Price System\& Resoure Allocation, Sixth Edition Oklahoma State University, The Dryden Press, 1976, p.173. 


\title{
AN ANALYTICAL STUDY OF THE CURRENT SITUATION OF THE PRODUCTION OF THE MAIN CITRUS VARIETIES IN EGYPT
}

\author{
SUAD ABDUL LATIF MOUSA MOHAMED and SHAHIRA MOHAMED REDA
}

Researchers at Agricultural Economics Research Institute- ARC.

(Manuscript received 5 April 2015)

\begin{abstract}
$\mathrm{C}$ itrus is the most Important Fruit in old Lands. The main objective of the research is to shed light on the production of citrus varieties in old lands to meet the limitation of land and water resources. The study showed that the total production of oranges in old lands increased by annual rate $1.24 \%$, that because the increasing in productivity per feddan annually which was than $1 \%$ during the period (2010-2012). The study also indicated that production of navel oranges increased in annual rate $2.9 \%$, that's because of the increasing in both the fruitful area, and the productivity per feddan in annual rate $1.94 \%$, and less than $1 \%$ respectively during the same period. This has proven to be statistical significant of this increase. The total cost of production of citrus per feddan in old lands estimated in about L.E 1404 thousand distributed between variable cost $74 \%$, and rent $26 \%$ during the period (2010-2012) wages of human labour about represented about $89 \%$, of the cost of agricultural labour which estimated in about L.E 4985 during the same period. Concerning the establishment new of farms of citrus in new lands is very costly which estimated about L.E 20 thousand per feddan. The net returns per feddan of citrus varieties were estimated in addalya lime about L.E9872, and sour lime about L.E 7694 during the period 2010-2012
\end{abstract}

The paper concluded with the following recommendations"

1- Reducing citrus varieties cultivated area which has not achieved the merit of productivity .

2- Expanding the cultivated area of citrus varieties which achieving high profit\& ability such as addalya lime governorates Bhera and Kafr-Elsheikh .

3- Reduce the cost of production of citrus per feddan in old lands by replacement machinery labour whith human labour.

4- the establishment new farms of citrus in the new lands ,by providing individual investors with loans from PBDAC or from the social fund for facing the high cost of production with suitable interest rate for increasing the production.

5- Issuing laws by the ministry of agriculture and ministry of irrigation to prevent planting the citrus varieties which achieved losses. because these citrus varieties need a high quantity of water and replacement of other agricultural rotation field crops, such as wheat and maize, and sugar beets which save water use. 